\title{
Equivalence of Continuous, Local and Infinitesimal Rigidity in Normed Spaces
}

\author{
Sean Dewar ${ }^{1}$ D \\ Received: 6 August 2018 / Revised: 2 August 2019 / Accepted: 28 August 2019 / \\ Published online: 20 September 2019 \\ (c) The Author(s) 2019
}

\begin{abstract}
We present a rigorous study of framework rigidity in general finite dimensional normed spaces from the perspective of Lie group actions on smooth manifolds. As an application, we prove an extension of Asimow and Roth's 1978/1979 result establishing the equivalence of local, continuous and infinitesimal rigidity for regular bar-and-joint frameworks in a $d$-dimensional Euclidean space. Further, we obtain upper bounds for the dimension of the space of trivial motions for a framework and establish the flexibility of small frameworks in general non-Euclidean normed spaces.
\end{abstract}

Keywords Bar-joint frameworks · Infinitesimal rigidity · Continuous rigidity $\cdot$ Local rigidity $\cdot$ Finite dimensional normed spaces

\section{Mathematics Subject Classification 52C25 · 52A21}

\section{Introduction}

A framework $(G, p)$ is an embedding $p$ of the vertices of a simple graph $G$ into a given normed space. With a given framework a natural question is whether it is in some sense "rigid". In Euclidean spaces many types of rigidity such as global, redundant and universal have been studied intensely $[8,12,26]$. We wish to detect whether a framework in a general normed space is structurally rigid in the sense that either any continuous motion of the vertices that preserves the edge lengths corresponds to an isometric motion of the embedded vertices (continuous rigidity) or that the embedding is locally unique up to an isometric map (local rigidity). In Euclidean space, one method is to consider the a priori stronger notion of infinitesimal rigidity as this implies local and continuous rigidity (see for example [7]). We shall prove the below theorem (found in Sect. 4):

\section{Editor in Charge: János Pach}

Sean Dewar

s.dewar@lancaster.ac.uk

1 Department of Mathematics and Statistics, Lancaster University, Lancaster LA1 4YF, UK 
Theorem 1.1 Let $(G, p)$ be a constant (see Sect. 4) finite framework in a finite dimensional normed space $X$, then the following are equivalent:

(i) $(G, p)$ is infinitesimally rigid in $X$,

(ii) $(G, p)$ is locally rigid in $X$,

(iii) $(G, p)$ is continuously rigid in $X$.

In 1978/79 L. Asimow and B. Roth proved Theorem 1.1 in the special case where $X$ is Euclidean [4,5]. Recently research has been undertaken into framework rigidity in non-Euclidean normed spaces, in particular spaces with $\ell_{p}$ norms $(p \in[1, \infty])$ [17], polyhedral norms [15] and matrix norms such as the Schatten $p$-norms [16]. For recent research into infinitesimal rigidity we refer the reader to $[13,25]$ for frameworks with symmetry, $[11,14]$ for infinitesimal rigidity concerning alternative types of frameworks, and [23] for frameworks on surfaces.

In Sect. 3 we will present a rigorous study of the orbit and the trivial motion space of a set of points. We will give an upper bound for the dimension of the space of trivial motions which will be achievable by most placements. Utilising this we shall in Sect. 4 prove Theorem 1.1.

In Sect. 5 we shall obtain further bounds on the dimension of the space of trivial infinitesimal motions for frameworks that lie on some hyperplane of the normed space. These results will allow us to prove that no small framework (a framework with less vertices than the dimension of the normed space plus one) on two or more vertices is infinitesimally rigid in a non-Euclidean space (see Theorem 5.8).

\section{Preliminaries}

All normed spaces $(X,\|\cdot\|)$ shall be assumed to be over $\mathbb{R}$ and finite dimensional; further we shall denote a normed space by $X$ when there is no ambiguity. For any normed space $X$ we shall use the notation $B_{r}(x), B_{r}[x]$ and $S_{r}[x]$ for the open ball, closed ball and the sphere with centre $x$ and radius $r>0$ respectively. We shall define a normed space to be a Euclidean space if its norm is generated by an inner product, otherwise $X$ is a non-Euclidean (normed) space.

Given normed spaces $X, Y$ we shall denote by $L(X, Y)$ the normed space of all linear maps from $X$ to $Y$ with the operator norm $\|\cdot\|_{\text {op }}$ and $A(X, Y)$ to be space of all affine maps from $X$ to $Y$ with the norm topology. If $X=Y$ we shall abbreviate to $L(X)$ and $A(X)$ and if $Y=\mathbb{R}$ with the standard norm we define $X^{*}:=L(X, \mathbb{R})$ and refer to the operator norm as $\|\cdot\|$ when there is no ambiguity. We denote by $\iota$ the identity map on $X$.

For a $C^{1}$-differentiable manifold $M$ we shall denote by $T_{x} M$ the tangent space of $M$ at $x \in M$ and $T M:=\bigcup_{x \in M} T_{x} M$. For a general reference on the theory of manifolds we refer the reader to [2, Sect. 3].

\subsection{Differentiation}

For normed spaces $X, Y$ and $U \subset X, V \subset Y$ we define a map $f: U \rightarrow V$ to be (Fréchet) differentiable at $x_{0} \in U^{\circ}$ (the interior of $U$ ) if there exists a linear map $d f\left(x_{0}\right): X \rightarrow Y$ such that 


$$
\frac{\left\|f\left(x_{0}+h\right)-f\left(x_{0}\right)-d f\left(x_{0}\right) h\right\|_{Y}}{\|h\|_{X}} \rightarrow 0
$$

as $h \rightarrow 0$; we refer to $d f\left(x_{0}\right)$ as the (Fréchet) derivative of $f$ at $x_{0}$. If $U^{\prime} \subset U^{\circ}$ is open and $f$ is differentiable at all points in $U^{\prime}$ we say that $f$ is differentiable on $U^{\prime}$. If $f$ is differentiable on $U^{\prime}$ and the map

$$
d f: U^{\prime} \rightarrow L(X, Y), x \mapsto d f(x)
$$

is continuous then we say that $f$ is $C^{1}$-differentiable on $U^{\prime}$ and define $d f$ to be the $C^{1}$-derivative of $f$; if $U^{\prime}=U$ we just say that $f$ is $C^{1}$-differentiable. For all $k \in \mathbb{N}$ we define inductively $d^{k} f:=d\left(d^{k-1} f\right)$ where $d^{1} f:=d f$ and $d^{0} f:=f$; by this we define $f$ to be $C^{k}$-differentiable if $d^{k} f$ exists and is continuous. If $f$ is $C^{k}$-differentiable for all $k \in \mathbb{N} \cup\{0\}$ we say $f$ is $C^{\infty}$-differentiable or smooth. If $f$ is $C^{k}$-differentiable and bijective with $C^{k}$-differentiable inverse we say that $f$ is a $C^{k}$-diffeomorphism or smooth diffeomorphism if $k=\infty$.

Some of the results referenced refer specifically to Gâteaux differentiation, however we will only consider Lipschitz maps between finite dimensional normed spaces and in this case Gâteaux differentiability is equivalent to differentiability by [6, Prop. 4.3]. If we were to observe the connection between the rigidity map and the rigidity operator of an infinite framework this would not hold to be true.

For $C^{k}$-manifolds $M$ and $N$ with $k \in \mathbb{N} \cup\{0, \infty\}$ we define a map $f: M \rightarrow N$ to be $C^{k}$-differentiable if for any $x \in M$ and chart $(V, \psi)$ of $N$ with $f(x) \in N$ there exists a chart $(U, \phi)$ of $M$ such that $x \in U, f(U) \subset V$ and $\psi \circ f \circ \phi^{-1}$ is $C^{k}$-differentiable on $U$. If $f$ also has a $C^{k}$-differentiable inverse then it is a $C^{k}$-diffeomorphism. For $k \geq 1$ we may define for each $x \in M$ the maps $d f(x): T_{x} M \rightarrow T_{f(x)} N$ and $d f: T M \rightarrow$ $T N$ that will be consistent if $M, N$ are normed spaces, we refer the reader to [2, Sect. 3.3] for more detail.

Remark 2.1 If we have a continuous path $\alpha:(a, b) \rightarrow X$ that is differentiable at $t \in(a, b)$ with differential $\alpha^{\prime}(t)$ in the traditional sence, i.e.,

$$
\alpha^{\prime}(t):=\lim _{h \rightarrow 0} \frac{\alpha(t+h)-\alpha(t)}{h}
$$

then $\alpha^{\prime}(t)=d \alpha(t)(1)$.

\subsection{Support Functionals, Smoothness and Strict Convexity}

Let $x \in X$ and $f \in X^{*}$, then we say that $f$ is support functional of $x$ if $\|f\|=\|x\|$ and $f(x)=\|x\|^{2}$. By an application of the Hahn-Banach theorem it can be shown that every point must have a support functional.

We say that a non-zero point $x$ is smooth if it has a unique support functional and define $\operatorname{smooth}(X) \subseteq X \backslash\{0\}$ to be the set of smooth points of $X$. If $\operatorname{smooth}(X) \cup\{0\}=X$ then we say that $X$ is smooth. We define a norm to be strictly convex if $\|t x+(1-t) y\|<$ 1 for all distinct $x, y \in S_{1}[0]$ and $t \in(0,1)$. 
The dual map of $X$ is the map $\varphi: \operatorname{smooth}(X) \cup\{0\} \rightarrow X^{*}$ that sends each smooth point to its unique support functional and $\varphi(0)=0$. It is immediate that $\varphi$ is homogeneous since $f$ is the support functional of $x$ if and only if $a f$ is the support functional of $a x$ for $a \neq 0$.

Remark 2.2 If $X$ is Euclidean with inner product $\langle\cdot, \cdot\rangle$ then all non-zero points are smooth and we have $\varphi(x)=\langle x, \cdot\rangle$ where $\langle x, \cdot\rangle: y \mapsto\langle x, y\rangle$.

Proposition 2.3 For any normed space $X$ the following properties hold:

(i) For $x_{0} \neq 0, x_{0} \in \operatorname{smooth}(X)$ if and only if $x \mapsto\|x\|$ is differentiable at $x_{0}$.

(ii) If $x \mapsto\|x\|$ is differentiable at $x_{0}$ then it has derivative $\frac{1}{\left\|x_{0}\right\|} \varphi\left(x_{0}\right)$.

(iii) The set $\operatorname{smooth}(X)$ is dense in $X$ and $\operatorname{smooth}(X)^{c}$ has measure zero with respect to the Lebesgue measure on $X$

(iv) The map $\varphi$ is continuous.

Proof (i) \& (ii) By [19, Lem. 1], $x \mapsto\|x\|$ is differentiable at $x_{0}$ if and only if $x_{0} \in \operatorname{smooth}(X)$ with derivative $\frac{1}{\left\|x_{0}\right\|} \varphi\left(x_{0}\right)$.

(iii) The result follows from (i) and [24, Thm. 25.5] as $x \mapsto\|x\|$ is convex.

(iv) As $\|\cdot\|$ is convex, by [24, Thm. 25.5], the map $x \mapsto \frac{1}{\|x\|} \varphi(x)$ is continuous on $\operatorname{smooth}(X)$; further, as $\|\cdot\|$ is continuous, $\varphi$ is continuous on $\operatorname{smooth}(X)$ also. Let $\left(x_{n}\right)_{n \in \mathbb{N}}$ be a sequence in $\operatorname{smooth}(X)$ that converges to 0 . As $\left\|\varphi\left(x_{n}\right)\right\|=\left\|x_{n}\right\|$ then $\left\|\varphi\left(x_{n}\right)\right\| \rightarrow 0$ as $n \rightarrow \infty$. As $\varphi(0)=0$ then $\varphi$ is continuous at $0 \in X$ also.

\subsection{Isometry Groups}

We shall define $\operatorname{Isom}(X,\|\cdot\|)$ to be the group of isometries of $(X,\|\cdot\|)$ and $\operatorname{Isom}^{\operatorname{Lin}}(X,\|\cdot\|)$ to be the group of linear isometries of $X$ with the group actions being composition; we shall denote these as $\operatorname{Isom}(X)$ and $\operatorname{Isom}^{\operatorname{Lin}}(X)$ if there is no ambiguity. It can be seen by Mazur-Ulam's theorem [27] that all isometries of a finite dimensional normed space are affine, i.e., each isometry is the unique composition of a linear isometry followed by a translation, thus $\operatorname{Isom}(X)$ has the topology inherited from $A(X)$. It follows from the Closed Subgroup theorem [2, Thm. 5.1.14] that for any normed space the group of isometries is a Lie group (a smooth finite dimensional manifold with smooth group operations) while the group of linear isometries is a compact Lie group since it is closed and bounded in $L(X)$.

Lemma 2.4 Let $X$ be a d-dimensional normed space, then the following holds:

(i) There exists a unique Euclidean space $\left(X,\|\cdot\|_{2}\right)$ such that $\operatorname{Isom}(X,\|\cdot\|)$ is a subgroup and a closed smooth submanifold of $\operatorname{Isom}\left(X,\|\cdot\|_{2}\right)$ and $\operatorname{Isom}^{\operatorname{Lin}}(X,\|\cdot\|)$ is a subgroup and a closed smooth submanifold of $\operatorname{Isom}^{\operatorname{Lin}}\left(X,\|\cdot\|_{2}\right)$.

(ii) If $X$ is Euclidean then:

(a) $\operatorname{dim} \operatorname{Isom}(X)=d(d+1) / 2$,

(b) $\operatorname{dim} \operatorname{Isom}^{\operatorname{Lin}}(X)=d(d-1) / 2$.

(iii) If $X$ is non-Euclidean then: 
(a) $d \leq \operatorname{dim} \operatorname{Isom}(X) \leq d(d-1) / 2+1$,

(b) $0 \leq \operatorname{dim} \operatorname{Isom}^{\operatorname{Lin}}(X) \leq(d-1)(d-2) / 2+1$.

Proof (i) This follows from [27, Cor. 3.3.4] and the Closed Subgroup theorem [2, Thm. 5.1.14].

(ii) See [10, Sect. 2.5.5] and [10, Sect. 2.5.9].

(iii) $[22$, Lem. 4].

\subsection{Placements and Bar-Joint Frameworks}

We shall assume that all graphs are simple, i.e., no loops or parallel edges, however we will allow them to have a countably infinite vertex set unless we explicitly state otherwise. We will denote $V(G)$ and $E(G)$ to be the vertex and edge sets of $G$ respectively. If $H$ is a subgraph of $G$ we will represent this by $H \subseteq G$. For a set $S$ we shall denote by $K_{S}$ the complete graph on the set $S$.

Let $X$ be a normed space. For any set $S$ we say $p \in X^{S}$ is a placement of $S$ in $X$; we will denote this $(p, S)$ if we need to clarify what set $p$ is the placement of. For a graph $G$ we say $p$ is a placement of $G$ in $X$ if $p$ is a placement of $V(G)$. We define a (bar-joint) framework to be a pair $(G, p)$ where $G$ is a graph and $p$ is a placement of $G$ in $X$. For all $X$ and $S$ we will gift $X^{S}$ the product topology from $X$; if $|S|<\infty$ we define the norm

$$
\|\cdot\|_{S}:\left(x_{v}\right)_{v \in S} \mapsto \max _{v \in S}\left\|x_{v}\right\|
$$

on $X^{S}$. For $x \in X^{S}$ and $T \subset S$ we define $\left.x\right|_{T}:=\left(x_{v}\right)_{v \in T} \in X^{T}$.

A placement $p$ is spanning in $X$ if the set $\left\{p_{v}: v \in S\right\}$ affinely spans $X$. A placement $p$ is in general position if for any choice of distinct vertices $v_{0}, v_{1}, \ldots, v_{n} \in S$ $(n \leq \operatorname{dim} X)$ the set $\left\{p_{v_{i}}: i=0,1, \ldots, n\right\}$ is affinely independent. It is immediate that if $p$ is in general position and $|S| \geq \operatorname{dim} X+1$ then $p$ is spanning. We denote the set of placements of $S$ in general position by $\mathcal{G}(S) \subseteq X^{S}$; likewise for any graph $G$ we let $\mathcal{G}(G):=\mathcal{G}(V(G))$. If $S$ is finite then $\mathcal{G}(S)$ is an open dense subset of $X^{S}$ and $X^{S} \backslash \mathcal{G}(S)$ has measure zero; we can see this as $\mathcal{G}(S)$ is the complement of an algebraic set.

For placements $(q, T),(p, S)$ we say $(q, T)$ is a subplacement of $(p, S)$ (or $(q, T) \subseteq(p, S))$ if $T \subseteq S$ and $p_{v}=q_{v}$ for all $v \in T$. For frameworks $(H, q)$ and $(G, p)$ we say $(H, q)$ is a subframework of $(G, p)$ (or $(H, q) \subseteq(G, p)$ ) if $H \subseteq G$ and $p_{v}=q_{v}$ for all $v \in V(H)$. If $H$ is also a spanning subgraph we say that $(H, q)$ is a spanning subframework of $(G, p)$.

\section{Trivial Motions of Placements}

\subsection{Structure of the Orbit of a Placement}

Let $\Gamma$ be a Lie group and $M$ a (finite dimensional) smooth manifold. If there exists a smooth group action 


$$
\phi: \Gamma \times M \rightarrow M,(g, x) \mapsto g \cdot x
$$

we say that $\phi$ is a Lie group action of $\Gamma$ on $M$. We define the following for all $x \in M$ :

(i) the stabiliser of $x, \operatorname{Stab}_{x}:=\{g \in \Gamma: g . x=x\}$,

(ii) the orbit of $x, \mathcal{O}_{x}:=\{g \cdot x: g \in \Gamma\}$,

(iii) $\phi_{x}: \Gamma \rightarrow \mathcal{O}_{x}, g \mapsto g . x$.

We say that $\Gamma$ acts properly on $M$ if the map

$$
\theta: \Gamma \times M \rightarrow M \times M,(g, x) \mapsto(\phi(g, x), x)
$$

is proper, i.e., the preimage of any compact set is compact. If $H$ is a closed subgroup of $\Gamma$ then by [2, Thm. 5.1.16] $\Gamma / H$ (the set of left cosets $g H, g \in \Gamma$ ) has a unique manifold structure such that the quotient map $\pi: \Gamma \rightarrow \Gamma / H$ is a smooth surjective submersion, i.e., $d \pi(g)$ is surjective for all $g \in \Gamma$.

Lemma 3.1 [1, Cor. 4.1.22] Let $\phi$ be a Lie group action of $\Gamma$ on M. Suppose $\Gamma$ acts properly on $M$, then $\mathcal{O}_{x}$ is a closed smooth submanifold of $M$ that is diffeomorphic to $\Gamma / \mathrm{Stab}_{x}$ under the map $\tilde{\phi}_{x}: g \mathrm{Stab}_{x} \mapsto g . x$.

For any set $S, x \in X^{S}$ and affine map $g \in A(X)$ we define $g . x:=\left(g\left(x_{v}\right)\right)_{v \in S \text {. }}$ With this notation we define for any $S$ the map

$$
\phi: \operatorname{Isom}(X) \times X^{S} \rightarrow X^{S}, x \mapsto g . x .
$$

If $|S|<\infty$ then this is a Lie group action of $\operatorname{Isom}(X)$ on $X^{S}$; we shall always refer to this group action if we mention $\operatorname{Isom}(X)$ acting on $X^{S}$.

Lemma 3.2 Let $(p, S)$ be a placement in $X$, then $\mathcal{O}_{p}$ is closed in $X^{S}$.

Proof Let $\left(q^{n}\right)_{n \in \mathbb{N}}$ be a convergent sequence in $\mathcal{O}_{p}$ with limit $q \in X^{S}$. Following from the definition of $\mathcal{O}_{p}$, for each $n \in \mathbb{N}$ there exist $g^{n} \in \operatorname{Isom}(X)$ such that $g^{n} \cdot p=q^{n}$. It follows from Mazur-Ulam's theorem [27] that for each $n \in \mathbb{N}$ there exist $G_{n} \in \operatorname{Isom}^{\operatorname{Lin}}(X)$ and $x_{n} \in X$ such that $g_{n}=T_{x_{n}} \circ G_{n}$, where $T_{x_{n}}$ is the translation map $y \mapsto y+x_{n}$.

As $\operatorname{Isom}^{\operatorname{Lin}}(X)$ is compact, there exists a convergent subsequence $\left(G_{n_{k}}\right)_{k \in \mathbb{N}}$ of $\left(G_{n}\right)_{n \in \mathbb{N}}$ with limit $G \in \operatorname{Isom}^{\operatorname{Lin}}(X)$. For any $v \in V(G)$,

$$
\left(q_{v}^{n_{k}}\right)_{k \in \mathbb{N}}=\left(G_{n_{k}}\left(p_{v}\right)+x_{n_{k}}\right)_{k \in \mathbb{N}}
$$

converges, thus $\left(x_{n_{k}}\right)_{k \in \mathbb{N}}$ converges also to some $x \in X$. If we define $q:=T_{x} \circ G$. $p \in$ $\mathcal{O}_{p}$ then $q$ is the limit of $\left(q^{n_{k}}\right)_{k \in \mathbb{N}}$. Since $\left(q^{n}\right)_{n \in \mathbb{N}}$ is a convergent sequence then it also has limit $q$ as required.

Lemma 3.3 For any $X$ and $|S|<\infty$ the group of isometries $\operatorname{Isom}(X)$ acts properly on $X^{S}$. 
Proof Let $\left(\left(g_{n} \cdot p^{n}, p^{n}\right)\right)_{n \in \mathbb{N}}$ be a convergent sequence in the image of $\theta$ : $\operatorname{Isom}(X) \times$ $X^{S} \rightarrow X^{S} \times X^{S}$ with limit $(q, p)$. It follows from Mazur-Ulam's theorem [27] that for each $n \in \mathbb{N}$ there exist $G_{n} \in \operatorname{Isom}^{\operatorname{Lin}}(X)$ and $x_{n} \in X$ such that $g_{n}=T_{x_{n}} \circ G_{n}$, where $T_{x_{n}}$ is the translation map $y \mapsto y+x_{n}$. As $\left(\left(g_{n} \cdot p^{n}, p^{n}\right)\right)_{n \in \mathbb{N}}$ converges then $\left(g_{n} \cdot p^{n}\right)_{n \in \mathbb{N}}$ and $\left(p^{n}\right)_{n \in \mathbb{N}}$ are bounded in $X^{S}$, thus $\left(x_{n}\right)_{n \in \mathbb{N}}$ is bounded as

$$
\left\|x_{n}\right\|=\left\|\left(x_{n}\right)_{v \in S}\right\|_{S} \leq\left\|G_{n} \cdot p^{n}+\left(x_{n}\right)_{v \in S}\right\|_{S}+\left\|G_{n} \cdot p^{n}\right\|_{S}=\left\|g_{n} \cdot p^{n}\right\|_{S}+\left\|p^{n}\right\|_{S} ;
$$

it follows by Bolzano-Weierstrass that we have a convergent subsequence $\left(x_{n_{k}}\right)_{k \in \mathbb{N}}$ with limit $x \in X$. Since $\operatorname{Isom}^{\operatorname{Lin}}(X)$ is compact, there exists a convergent subsequence $\left(G_{n_{k_{l}}}\right)_{l \in \mathbb{N}}$ of $\left(G_{n_{k}}\right)_{k \in \mathbb{N}}$ with limit $G \in \operatorname{Isom}^{\operatorname{Lin}}(X)$; this implies $\left(g_{n_{k_{l}}}\right)_{l \in \mathbb{N}}$ converges to $g:=T_{x} \circ G$. As $\left(\left(g_{n}, p^{n}\right)\right)_{n \in \mathbb{N}}$ has a convergent subsequence it follows that $\theta$ is proper as required.

Lemma 3.4 Let $p$ be a placement of a finite set in $X$, then $\mathcal{O}_{p}$ is a closed smooth submanifold of $X^{V(G)}$ and the map

$$
\tilde{\phi}_{p}: \operatorname{Isom}(X) / \operatorname{Stab}_{p} \rightarrow \mathcal{O}_{p}, g \operatorname{Stab}_{p} \mapsto g \cdot p
$$

is a smooth diffeomorphism.

Proof By Lemmas 3.3 and 3.1 it follows that $\mathcal{O}_{p}$ is a closed smooth submanifold of $X^{V(G)}$ diffeomorphic to $\operatorname{Isom}(X) / \operatorname{Stab} p$ under the diffeomorphism $\tilde{\phi}_{p}$.

Lemma 3.5 Let $(q, T) \subset(p, S)$ be placements in $X$ where the affine span of $\left\{p_{v}\right.$ : $v \in S\}$ is equal to the affine span of $\left\{q_{v}: v \in T\right\}$. If $|T|<\infty$ then $\mathcal{O}_{p}$ is a smooth manifold that is diffeomorphic to $\mathcal{O}_{q}$ and the restriction map

$$
\rho: \mathcal{O}_{p} \rightarrow \mathcal{O}_{q},\left(x_{v}\right)_{v \in S} \mapsto\left(x_{v}\right)_{v \in T}
$$

is a smooth diffeomorphism.

Proof Define the (finite dimensional) linear spaces

$$
A_{p}:=\{h . p: h \in A(X)\}, \quad A_{q}:=\{h . q: h \in A(X)\} .
$$

We note that the linear map $\tilde{\rho}: A_{p} \rightarrow A_{q}$ where $\tilde{\rho}(x):=\left.x\right|_{T}$ is a continuous linear isomorphism. This implies the map $\left.\tilde{\rho}^{-1}\right|_{\mathcal{O}_{q}}$ is a smooth embedding into $A_{p}$ with image $\mathcal{O}_{p}$. By Lemma 3.4, $\mathcal{O}_{q}$ is a smooth manifold, thus $\mathcal{O}_{q}$ is diffeomorphic to $\mathcal{O}_{p}$ and $\rho:=\left.\tilde{\rho}\right|_{\mathcal{O}_{p}} ^{\mathcal{O}_{q}}$ is a smooth diffeomorphism.

We will define a continuous path through a placement $p$ in $X^{S}$ to be a family $\alpha:=\left(\alpha_{v}\right)_{v \in S}$ of continuous paths $\alpha_{v}:(-\delta, \delta) \rightarrow X$ (for some fixed $\delta>0$ ) where $\alpha_{v}(0)=p_{v}$ for all $v \in S$. If $\alpha(t):=\left(\alpha_{v}(t)\right)_{v \in S} \in \mathcal{O}_{p}$ for all $t \in(-\delta, \delta)$ then $\alpha$ is a trivial finite motion.

Let $u \in X^{S}$. If there exists a trivial finite motion $\alpha$ of $p$ that is differentiable at $t=0$ and $u_{v}=\alpha_{v}^{\prime}(0)$ for all $v \in S$ then we say that $u$ is a trivial (infinitesimal) motion of 
$p$. For any placement $p$ we shall denote $\mathcal{T}(p)$ to be the the set all trivial infinitesimal motions of $p$.

Theorem 3.6 Let $p$ be a placement in $X$, then $\mathcal{O}_{p}$ is a smooth manifold with tangent space $\mathcal{T}(p)$ at $p$ and

$$
\tilde{\phi}_{p}: \operatorname{Isom}(X) / \operatorname{Stab}_{p} \rightarrow \mathcal{O}_{p}, g \operatorname{Stab}_{p} \mapsto g \cdot p
$$

is a smooth diffeomorphism.

Proof Choose a finite subplacement $(q, T)$ of $(p, S)$ so that the $p$ and $q$ affinely span the same space, then by Lemma $3.4, \mathcal{O}_{q}$ is a smooth manifold diffeomorphic to $\operatorname{Isom}(X) / \operatorname{Stab}_{q}$ under the smooth diffeomorphism $\tilde{\phi}_{q}$. By Lemma 3.5, $\mathcal{O}_{p}$ is a smooth manifold diffeomorphic to $\operatorname{Isom}(X) / \operatorname{Stab}_{p}$ and the restriction map $\rho: \mathcal{O}_{p} \rightarrow \mathcal{O}_{q}$ is a smooth diffeomorphism. As $\tilde{\phi}_{p}=\rho^{-1} \circ \tilde{\phi}_{q}$ then it is also a smooth diffeomorphism. It follows from its definition that $\mathcal{T}(p)$ is the tangent space of $\mathcal{O}_{p}$ at $p$.

Corollary 3.7 The map $\phi_{p}$ is a smooth submersion and $d \phi_{p}(\iota): T_{\iota} \operatorname{Isom}(X) \rightarrow \mathcal{T}(p)$ is surjective with $d \phi_{p}(\iota) g=g$.p for all $g \in T_{\iota} \operatorname{Isom}(X)$. Further, $\operatorname{ker} d \phi_{p}(\iota)=T_{\iota} \operatorname{Stab}_{p}$.

Proof By Theorem 3.6, $\tilde{\phi}_{p}$ is a smooth diffeomorphism. We note that $\phi_{p}=\tilde{\phi}_{p} \circ \pi$ where $\pi: \operatorname{Isom}(X) \rightarrow \operatorname{Isom}(X) / \operatorname{Stab}_{p}$ is the natural quotient map. By the Closed Subgroup theorem [2, Thm. 5.1.14], $\pi$ is a smooth submersion, thus $\phi_{p}$ is a smooth submersion also and $d \phi_{p}(\iota)$ is surjective. As $\tilde{\phi}_{p}$ is a smooth diffeomorphism then $\operatorname{ker} d \phi_{p}(\iota)=\operatorname{ker} \pi$ as required.

Corollary 3.8 Let $(p, S)$ and $(q, T)$ be placements in $X$ where the affine span of $\left\{p_{v}\right.$ : $v \in S\}$ is equal to the affine span of $\left\{q_{v}: v \in T\right\}$, then the following hold:

(i) The orbits $\mathcal{O}_{p}$ and $\mathcal{O}_{q}$ are diffeomorphic.

(ii) $\operatorname{dim} \mathcal{T}(p)=\operatorname{dim} \mathcal{T}(q)$.

(iii) If $(q, T) \subseteq(p, S)$ then the restriction map

$$
\rho: \mathcal{O}_{p} \rightarrow \mathcal{O}_{q},\left(x_{v}\right)_{v \in S} \mapsto\left(x_{v}\right)_{v \in T}
$$

is a smooth diffeomorphism.

Proof (iii) Choose a finite subplacement $(r, U) \subseteq(q, T) \subseteq(p, S)$ so that the affine span of $\left\{r_{v}: v \in U\right\}$ is equal to the affine span of $\left\{q_{v}: v \in T\right\}$. Define the restriction maps $\rho_{T}: \mathcal{O}_{q} \rightarrow \mathcal{O}_{r}$ and $\rho_{S}: \mathcal{O}_{p} \rightarrow \mathcal{O}_{r}$, then by Lemma 3.5, $\rho_{T}, \rho_{S}$ are smooth diffeomorphisms. As $\rho=\rho_{T}^{-1} \circ \rho_{S}$ then it is also a smooth diffeomorphism.

(i) If $(q, T) \subseteq(p, S)$ this follows from (iii). Suppose $(q, T)$ is not a subplacement of $(p, S)$. Define $(t, S \sqcup T)$ to be the placement where $\left.t\right|_{S}=p$ and $\left.t\right|_{T}=q$. We note all three placements have the same affine span of their placement points. As $(p, S) \subset(t, S \sqcup T)$ and $(q, T) \subseteq(t, S \sqcup T)$ then by (iii) we have $\mathcal{O}_{p} \cong \mathcal{O}_{t} \cong \mathcal{O}_{q}$ as required.

(ii) By Theorem 3.6, $\mathcal{O}_{p}$ has tangent space $\mathcal{T}(p)$ at $p$ and $\mathcal{O}_{q}$ has tangent space $\mathcal{T}(q)$ at $q$. By (i), $\mathcal{O}_{p} \cong \mathcal{O}_{q}$ and so $\operatorname{dim} \mathcal{T}(p)=\operatorname{dim} \mathcal{T}(q)$. 


\subsection{Upper and Lower Bounds of the Dimension of $\mathcal{T}(p)$}

Proposition 3.9 The following hold for any placement $p$ :

(i) $d \phi_{p}(\iota)$ is injective if and only if $\phi_{p}$ is a smooth local diffeomorphism, i.e., $d \phi_{p}(g)$ is bijective for all $g \in \operatorname{Isom}(X)$.

(ii) $\phi_{p}$ is injective if and only if $\phi_{p}$ is a smooth diffeomorphism.

If either (i) or (ii) hold then $\operatorname{dim} \mathcal{T}(p)=\operatorname{Isom}(X)$.

Proof If $\phi_{p}$ is a local diffeomorphism it follows $d \phi_{p}(\iota)$ is bijective. Suppose $d \phi_{p}(\iota)$ is injective. Choose $g \in \operatorname{Isom}(X)$, then $\operatorname{dim} \operatorname{ker} d \phi_{p}(g)=0$; this follows as there exists non-zero $u \in \operatorname{ker} d \phi_{p}(g)$ with corresponding smooth curve $\alpha:(-1,1) \rightarrow \operatorname{Isom}(X)$ (i.e., $\alpha(0)=g, \alpha^{\prime}(0)=u$ ) then the curve $t \mapsto g^{-1} \alpha(t)$ generates a non-zero tangent vector at $\iota$ that lies in the kernel of $d \phi_{p}(\iota)$. By this it follows that $d \phi_{p}(\iota)$ is injective if and only if $d \phi_{p}(g)$ is injective for all $g \in \operatorname{Isom}(X)$. By Corollary 3.7 it follows that $d \phi_{p}(g)$ is bijective for all $g \in \operatorname{Isom}(X)$ and so is a local diffeomorphism.

If $\phi_{p}$ is injective then $\operatorname{Stab}_{p}$ is trivial, thus $\operatorname{Isom}(X) / \operatorname{Stab}_{p}=\operatorname{Isom}(X)$ and $\tilde{\phi}_{p}=$ $\phi_{p}$. By Theorem 3.6 it then follows $\phi_{p}$ is a smooth diffeomorphism. Conversely suppose $\phi_{p}$ is a smooth diffeomorphism, then as $\phi_{p}=\tilde{\phi}_{p} \circ \pi$ it follows that the quotient map $\pi$ is a diffeomorphism. This implies $\pi$ is a group isomorphism, thus $\mathrm{Stab}_{p}$ is trivial and $\phi_{p}$ is injective.

If either (i) or (ii) hold then $d \phi_{p}(\iota)$ is bijective and $\operatorname{dim} \mathcal{T}(p)=\operatorname{Isom}(X)$.

Definition 3.10 We define a placement $p$ to be full if $\phi_{p}$ is a local diffeomorphism and isometrically full if $\phi_{p}$ is a diffeomorphism.

It is immediate that any isometrically full placement is full. By Proposition 3.9 (i), our notion of full agrees with that given in [16]. The set of full placements of a set $S$ will be denoted by $\operatorname{Full}(S)$ and likewise the set of full placements of a graph $G$ will be denoted by $\operatorname{Full}(G)$.

Corollary 3.11 All spanning placements are isometrically full.

Proof Suppose $g \cdot p=p$ and choose $v_{0}, \ldots, v_{d} \in S$ so that $p_{v_{0}}, \ldots, p_{v_{d}}$ is an affine basis of $X$, then $g\left(p_{v_{i}}\right)=p_{v_{i}}$ for all $i=0, \ldots, d$. By Mazum-Ulam's theorem [27], $g$ is affine and so since $p_{v_{0}}, \ldots, p_{v_{d}}$ is an affine basis of $X$ this map must be unique. As $\iota . p=p$ then $g=\iota$ and $\phi_{p}$ is injective. The result now follows by Proposition 3.9 (ii).

Example 3.12 We shall denote by $X$ the space $\mathbb{R}^{2}$ with the $\ell_{3}$-norm. The linear isometries of $X$ are generated by the $\pi / 2$ anticlockwise rotation around the origin and the reflection in the line $\{(t, 0): t \in \mathbb{R}\}$. Let $S=\left\{v_{1}, v_{2}\right\}$ and $(p, S)$ and $(q, S)$ be the nonspanning placements in $X$ where $p_{v_{1}}=q_{v_{1}}=0, p_{v_{2}}=(1,0)$ and $q_{v_{2}}=(1,2)$. Both placements are full in $X$, however $q$ is isometrically full while $p$ is not. This example shows that while all spanning placements are isometrically full and all isometrically full placements are full the reverse is not necessarily true.

Proposition 3.13 Let $d+1 \leq|S|<\infty$ and $\operatorname{dim} X=d$. Then Full $(S)$ is an open dense subset of $X^{S}$ and Full $(S)^{c}$ has measure zero with respect to the Lebesgue measure on $X^{S}$. 
Proof Since $|S| \geq d+1$ then all placements in general position are spanning. By Corollary 3.11 we have $\mathcal{G}(S) \subset \operatorname{Full}(S)$, thus as $\mathcal{G}(S)$ is dense in $X^{S}$ then $\operatorname{Full}(S)$ is dense in $X^{S}$. Since $\mathcal{G}(S)^{c}$ is an algebraic set then it has measure zero, thus it follows $\operatorname{Full}(S)^{c}$ also has measure zero.

Define the affine map

$$
F: X^{S} \rightarrow L\left(T_{\iota} \operatorname{Isom}(X), X^{S}\right), p \mapsto d \phi_{p}(\iota)
$$

The set of injective maps of $L\left(T_{l} \operatorname{Isom}(X), X^{S}\right)$ is open. We note Full $(S)$ is the preimage of the set of injective maps of $L\left(T_{\iota} \operatorname{Isom}(X), X^{S}\right)$ under $F$ by Proposition 3.9 (ii) and so $\operatorname{Full}(S)$ is open.

Corollary 3.14 All isometrically full placements in $X$ are spanning if and only if $X$ is Euclidean.

Proof Suppose all isometrically full placements in $X$ are spanning, then it follows by Proposition 3.9 (ii) that for all linear hyperplanes $Y$ of $X$ there exists a linear map $T_{Y} \neq \iota$ that is invariant on $Y$. By [3, (4.7)], $X$ is Euclidean.

Conversely suppose $X$ is Euclidean. If $p$ is a non-spanning placement then $p$ lies in some affine hyperplane $H$. We note that if $h$ is the reflection in $H$ then $h \cdot p=p$, thus by Proposition 3.9(ii) $p$ is not isometrically full.

We may now give an upper and lower bound for the dimension of $\mathcal{T}(p)$.

Theorem 3.15 For any placement $p$ in a d-dimensional space $X$,

$$
d \leq \operatorname{dim} \mathcal{T}(p) \leq \operatorname{dim} \operatorname{Isom}(X)
$$

with $\operatorname{dim} \mathcal{T}(p)=\operatorname{dim} \operatorname{Isom}(X)$ if and only if $p$ is full.

Proof By Corollary 3.7, $d \phi_{p}(\iota): T_{\iota} \operatorname{Isom}(X) \rightarrow \mathcal{T}(p)$ is surjective, thus $\operatorname{dim} \mathcal{T}(p) \leq$ $\operatorname{dim} \operatorname{Isom}(X)$. Let $x_{1}, \ldots, x_{d} \in X$ be a basis and define for each $i \in\{1, \ldots, d\}$ the trivial finite flex $\alpha(i)$ where for each $v \in S$ we have $\alpha(i)_{v}:(-1,1) \rightarrow X$, $t \mapsto p_{v}+t x_{i}$. We note that $\left(\alpha(i)_{v}^{\prime}(0)\right)_{v \in S}=\left(x_{i}\right)_{v \in S} \in \mathcal{T}(p)$ for each $i \in\{1, \ldots, d\}$, thus $\operatorname{dim} \mathcal{T}(p) \geq d$.

If $p$ is full then by Proposition 3.9, $\operatorname{dim} \mathcal{T}(p)=\operatorname{dim} \operatorname{Isom}(X)$. If $\operatorname{dim} \mathcal{T}(p)=$ $\operatorname{dim} \operatorname{Isom}(X)$ then by Corollary $3.7, d \phi_{p}(\iota)$ is bijective; it then follows by Proposition 3.9 (i) that $p$ is full.

A rigidmotion of $X$ is a family $\gamma:=\left(\gamma_{x}\right)_{x \in X}$ of continuous maps $\gamma_{x}:(-\delta, \delta) \rightarrow X$, $x \in X$ (for some fixed $\delta>0$ ) where $\gamma_{x}(0)=x$ and $\left\|\gamma_{x}(t)-\gamma_{y}(t)\right\|=\|x-y\|$ for all $x, y \in X$ and $t \in(-\delta, \delta)$. The following shows that our definition of a trivial finite motion agrees with the definition given in [17] if a framework is isometrically full.

Proposition 3.16 Let $p$ be an isometrically full placement in X. If $\alpha$ is a continuous path through $p$ in $X^{S}$ then the following are equivalent:

(i) $\alpha$ is a trivial finite motion. 
(ii) There exists a unique continuous path $h:(-\delta, \delta) \rightarrow \operatorname{Isom}(X)$ such that $h_{t}\left(p_{v}\right)=$ $\alpha_{v}(t)$ for all $t \in(-\delta, \delta), v \in S$.

(iii) There exists a unique rigid motion $\gamma$ such that $\gamma_{p_{v}}=\alpha_{v}$ for all $v \in S$.

Proof (i) $\Rightarrow$ (ii) As $\alpha$ is a continuous path in $\mathcal{O}_{p}$ and $\phi_{p}$ is a smooth diffeomorphism we define the unique continuous path $h:=\phi_{p}^{-1} \circ \alpha$.

(ii) $\Rightarrow$ (iii) Define $\gamma$ to be the unique family of maps $\gamma$ where $\gamma_{x}(t)=h_{t}(x)$ for all $x \in X$ and $t \in(-\delta, \delta)$, then $\gamma$ is a rigid motion as required.

(iii) $\Rightarrow$ (i) We note that $\gamma$ restricted to the set $\left\{p_{v}: v \in S\right\}$ is a trivial finite motion.

\section{Equivalence of Continuous, Local and Infinitesimal Rigidity}

\subsection{Continuous, Local and Infinitesimal Rigidity}

We say that an edge $v w \in E(G)$ of a framework $(G, p)$ is well-positioned if $p_{v}-p_{w} \in$ $\operatorname{smooth}(X)$; if this holds we define $\varphi_{v, w}:=\varphi\left(\frac{p_{v}-p_{w}}{\left\|p_{v}-p_{w}\right\|}\right)$. If all edges of $(G, p)$ are wellpositioned we say that $(G, p)$ is well-positioned and $p$ is a well-positioned placement of $G$. We shall denote the subset of well-positioned placements of $G$ in $X$ by the set $\mathcal{W}(G)$.

Lemma 4.1 Let $G$ be finite, then $\mathcal{W}(G)$ a dense subset of $X^{V(G)}$ and $\mathcal{W}(G)^{c}$ has measure zero with respect to the Lebesgue measure on $X^{V(G)}$.

Proof By Proposition 2.3(iii), the set $\operatorname{smooth}(X)$ is dense and its compliment has measure zero, thus the result holds for all graphs with a single edge. Suppose the result holds for all graphs with $n-1$ edges and let $G$ be any graph with $n$ edges. Choose $v w \in E(G)$, and define $G_{1}, G_{2}$ to be the graphs on $V(G)$ where $E\left(G_{1}\right)=E(G) \backslash\{v w\}$ and $E\left(G_{2}\right)=\{v w\}$ then $\mathcal{W}\left(G_{1}\right)^{c}$ and $\mathcal{W}\left(G_{2}\right)^{c}$ have measure zero by assumption. As $\mathcal{W}(G)^{c}=\mathcal{W}\left(G_{1}\right)^{c} \cup \mathcal{W}\left(G_{2}\right)^{c}$ then $\mathcal{W}(G)^{c}$ has measure zero also; this further implies $\mathcal{W}(G)$ is also dense. The result now follows by induction.

We define the rigidity map of $G$ (in $X$ ) to be the continuous map

$$
f_{G}: X^{V(G)} \rightarrow \mathbb{R}^{E(G)}, x=\left(x_{v}\right)_{v \in V(G)} \mapsto\left(\left\|x_{v}-x_{w}\right\|\right)_{v w \in E(G)}
$$

and for well-positioned placements $p$ we also define the rigidity operator of $G$ at $p$ in $X$ to be the continuous linear map

$$
d f_{G}(p): X^{V(G)} \rightarrow \mathbb{R}^{E(G)}, x=\left(x_{v}\right)_{v \in V(G)} \mapsto\left(\varphi_{v, w}\left(x_{v}-x_{w}\right)\right)_{v w \in E(G)}
$$

For any framework we define the configuration space of $(G, p)$ in $X$ to be the set $f_{G}^{-1}\left[f_{G}(p)\right]$.

Proposition 4.2 [19, Prop. 6] If $G$ is finite then $f_{G}$ is differentiable at $p$ if and only if $p$ is a well-positioned placement of $G$; if this holds then the rigidity operator at $p$ is the derivative of the rigidity map at $p$. 
Lemma 4.3 If $G$ is finite then the map

$$
d f_{G}: \mathcal{W}(G) \rightarrow L\left(X^{V(G)}, \mathbb{R}^{E(G)}\right), x \mapsto d f_{G}(x)
$$

is continuous.

Proof This follows from Proposition 2.3 (iv).

For a finite graph $G$ we say that a well-positioned framework $(G, p)$ is regular if for all $q \in \mathcal{W}(G)$ we have rank $d f_{G}(p) \geq \operatorname{rank} d f_{G}(q)$. We shall denote the subset of $\mathcal{W}(G)$ of regular placements of $G$ by $\mathcal{R}(G)$.

Lemma 4.4 Let $G$ be finite, then $\mathcal{R}(G)$ is a non-empty open subset of $\mathcal{W}(G)$.

For this lemma we shall need to use the fact that the rank function on the space of linear maps between finite dimensional normed spaces $X, Y$ is lower semi-continuous, i.e., for all $c \geq 0$ the set $\{T \in L(X, Y): \operatorname{rank} T \geq c\}$ is open.

Proof Let $n:=\sup \left\{\operatorname{rank} d f_{G}(p): p \in \mathcal{W}(G)\right\}$. The $\operatorname{rank}$ function $T \mapsto \operatorname{rank} T$ is lower semi-continuous and by Lemma $4.3, d f_{G}$ is continuous, thus the map

$$
f: \mathcal{W}(G) \rightarrow \mathbb{N}, p \mapsto \operatorname{rank} d f_{G}(p)
$$

is lower semi-continuous. As $\mathcal{R}(G)=f^{-1}[[n, \infty)]$ then $\mathcal{R}(G)$ is open.

We define a finite flex of a framework $(G, p)$ to be a continuous path $\alpha$ through a placement $p$ where $\left\|\alpha_{v}(t)-\alpha_{w}(t)\right\|=\left\|p_{v}-p_{w}\right\|$ for all $v w \in E(G)$ and $t \in(-\delta, \delta)$. If $\alpha$ is a trivial finite motion of a placement $p$ of $G$ we say $\alpha$ is a trivial finite flex of $(G, p)$; we note that $\alpha$ will automatically be a finite flex of $(G, p)$ as isometries preserve distance.

If the only finite flexes of $(G, p)$ are trivial then $(G, p)$ is continuously rigid (in $X) ;(G, p)$ will be defined to be continuously flexible if it is not continuously rigid. For a finite framework $(G, p)$ we say $(G, p)$ is locally rigid (in $X)$ if there exists a neighbourhood $U \subseteq X^{V(G)}$ of $p$ such that $f_{G}^{-1}\left[f_{G}^{-1}(p)\right] \cap U=\mathcal{O}_{p} \cap U$; likewise we shall define a framework to be locally flexible if it is not locally rigid. We classify these as types of finite rigidity.

The following result proves that our definition for "continuous flexibility" is equivalent to the definition of "flexibility" given in [4].

Proposition 4.5 Let $(G, p)$ be a framework in X. If $(G, p)$ is continuously flexible then there exists a finite flex $\alpha:=\left(\alpha_{v}\right)_{v \in V(G)}$ with $\alpha_{v}:(-\delta, \delta) \rightarrow X$ for all $v \in V(G)$, such that $\alpha(t) \notin \mathcal{O}_{p}$ for all $t \neq 0$.

Proof Suppose $(G, p)$ is continuously flexible, then there exists a non-trivial finite flex $\beta:(-r, r) \rightarrow X^{V(G)}$ for all $v \in V(G)$; we note that $\beta$ is continuous by definition. Let $A:=f_{G}^{-1}\left[f_{G}(p)\right] \backslash \mathcal{O}_{p}$. By Lemma 3.2, $A$ is an open subset of $f_{G}^{-1}\left[f_{G}(p)\right]$, thus $\beta^{-1}[A]$ is an open set in $(-r, r)$. Any open set of $\mathbb{R}$ is the countable union of disjoint 
open intervals, thus there exists $(a, b) \subset \beta^{-1}[A]$ where (without loss of generality) we may assume $a \notin \beta^{-1}[A]$.

As $\beta(a) \in \mathcal{O}_{p}$, choose $g \in \operatorname{Isom}(X)$ such that $g \cdot p=\beta(a)$. We now define $\alpha$ to be the finite flex where $\delta:=b-a$, and

$$
\alpha(t):= \begin{cases}g^{-1} \cdot \beta(t+a) & \text { if } t \geq 0 \\ g^{-1} \cdot \beta(-t+a) & \text { if } t<0\end{cases}
$$

We define $u \in X^{V(G)}$ to be a trivial (infinitesimal) flex of $(G, p)$ if $u$ is a trivial motion of $p$. If $(G, p)$ is well-positioned we say that $u \in X^{V(G)}$ is an (infinitesimal) flex of $(G, p)$ if $d f_{G}(p) u=0$. The following proposition shows a link between finite and infinitesimal flexes for frameworks.

Lemma 4.6 Let $(G, p)$ be a well-positioned framework in $X$ and $\alpha$ a finite flex of $(G, p)$ that is differentiable at 0 , then $\left(\alpha_{v}^{\prime}(0)\right)_{v \in V(G)}$ is an infinitesimal flex of $(G, p)$.

Proof This follows from the proof of [17, Lem. 2.1(ii)].

Since all trivial flexes of $(G, p)$ are trivial motions of $p$ we shall also denote $\mathcal{T}(p)$ to be the set all trivial infinitesimal flexes $(G, p)$. If $(G, p)$ is well-positioned we define $\mathcal{F}(G, p)$ to be the space of all infinitesimal flexes of $(G, p)$. The latter is clearly a linear space as it is exactly the kernel of the rigidity operator. By Lemma 4.6, it follows $\mathcal{T}(p) \subseteq \mathcal{F}(G, p)$.

A well-positioned framework $(G, p)$ is infinitesimally rigid (in $X)$ if every flex is trivial and infinitesimally flexible (in $X$ ) otherwise. We shall define a well-positioned $(G, p)$ framework to be independent if the rigidity operator of $G$ at $p, d f_{G}(p)$, is surjective (or equivalently, if $G$ is finite, $\left.|E(G)|=\operatorname{rank} d f_{G}(p)\right)$ and define $(G, p)$ to be dependent otherwise. If a framework is infinitesimally rigid and independent we shall say that it is isostatic. We shall use the convention that any framework with no edges (regardless of placement) is independent and that $\left(K_{1}, p\right)$ is isostatic for any choice of placement $p$. It is immediate that if a framework is independent then its placement is regular, however the reverse does not necessarily hold.

Remark 4.7 In the setting of Euclidean space, all of the above definitions agree with those used in $[4,5]$.

Example 4.8 Let $G$ be a (possibly infinite) tree with vertex set $\left\{v_{1}, v_{2}, \ldots\right\}$ and wellpositioned placement $p$. By possibly reordering, we shall assume that for every $n \in \mathbb{N}$, the vertex $v_{n}$ is connected to exactly one vertex $v_{i} \in\left\{v_{1}, \ldots, v_{n-1}\right\}$ by an edge. Choose any $a \in \mathbb{R}^{E(G)}$. We define $u \in X^{V(G)}$ inductively by setting $u_{v_{1}}=0$ and choosing $u_{v_{n}}$ such that $\varphi_{v_{n}, v_{i}}\left(u_{v_{n}}-u_{v_{i}}\right)=a_{v_{n}, v_{i}}$ for the unique vertex $v_{i} \in\left\{v_{1}, \ldots, v_{n-1}\right\}$ connected to $v_{n}$ by an edge. As $d f_{G}(p)(u)=a,(G, p)$ is independent. If we chose $G$ to be a finite tree, $(G, p)$ would be regular also.

Lemma 4.9 Let $(G, p)$ be a finite (possibly not spanning) framework in a $d$ dimensional normed space $X$ with $|V(G)| \geq d+1$. Suppose $q \in \mathcal{R}(G)$ is full, then the following hold: 
(i) If $(G, p)$ is independent then $(G, p)$ is regular and $(G, q)$ is independent.

(ii) If $(G, p)$ is infinitesimally rigid then $(G, p)$ is regular, $p$ is full and $(G, q)$ is infinitesimally rigid.

Proof (i) As $(G, p)$ is independent then $d f_{G}(p)$ is surjective. As surjective linear maps have maximal possible rank then $(G, p)$ is regular. Since $q$ is regular it follows that $(G, q)$ is independent.

(ii) As $(G, q)$ is regular then by the Rank-Nullity theorem we have

$$
d|V(G)|-\operatorname{dim} \mathcal{T}(p)=\operatorname{rank} d f_{G}(p) \leq \operatorname{rank} d f_{G}(q) \leq d|V(G)|-\operatorname{dim} \mathcal{T}(q),
$$

thus by Theorem 3.15, $\operatorname{dim} \mathcal{T}(q) \leq \operatorname{dim} \mathcal{T}(p) \leq \operatorname{dim} \operatorname{Isom}(X)$. As $q$ is full then by Theorem 3.15, $\operatorname{dim} \mathcal{T}(q)=\operatorname{dim} \operatorname{Isom}(X)$. It follows that $\operatorname{dim} \mathcal{T}(p)=\operatorname{dim} \operatorname{Isom}(X)$ and thus $p$ is full. From the inequality it also follows that $(G, q)$ is infinitesimally rigid.

Lemma 4.10 Let $(G, p)$ be a independent framework and $(H, q) \subset(G, p)$, then $(H, q)$ is also independent.

Proof Choose $a \in \mathbb{R}^{E(H)}$. As $a \times(0)_{v w \in E(G) \backslash E(H)} \in \mathbb{R}^{E(G)}$ and $(G, p)$ is independent there exists $x \in X^{V(G)}$ such that $d f_{G}(p)(x)=a \times(0)_{v w \in E(G) \backslash E(H)}$. We now note $d f_{H}(q)\left(\left.x\right|_{V(H)}\right)=a$ as required.

The following gives us some necessary and sufficient conditions for infinitesimal rigidity.

Theorem 4.11 [19, Thm. 10] Let finite $(G, p)$ be well-positioned in X, then the following hold:

(i) If $(G, p)$ is independent then $|E(G)|=(\operatorname{dim} X)|V(G)|-\operatorname{dim} \mathcal{F}(G, p)$.

(ii) If $(G, p)$ is infinitesimally rigid then $|E(G)| \geq(\operatorname{dim} X)|V(G)|-\operatorname{dim} \mathcal{T}(p)$.

The following gives an equivalence for isostaticity.

Proposition 4.12 Let $(G, p)$ be a well-positioned framework in $X$. If any two of the following properties hold then so does the third (and $(G, p)$ is isostatic):

(i) $|E(G)|=(\operatorname{dim} X)|V(G)|-\operatorname{dim} \mathcal{T}(p)$,

(ii) $(G, p)$ is infinitesimally rigid,

(iii) $(G, p)$ is independent.

Proof Apply the Rank-Nullity theorem to the rigidity operator of $G$ at $p$. The result follows the same method as [9, Lem. 2.6.1.c].

Using the results from the last section we may now give a stronger result for independent frameworks.

Corollary 4.13 Let $(G, p)$ be a finite independent framework with $|V(G)| \geq \operatorname{dim} X+1$. Then for all $H \subset G$ with $|V(H)| \geq d+1$ we have $|E(H)| \leq(\operatorname{dim} X)|V(H)|-$ $\operatorname{dim} \operatorname{Isom}(X) . I f(G, p)$ is also isostatic then $|E(G)|=(\operatorname{dim} X)|V(G)|-\operatorname{dim} \operatorname{Isom}(X)$. 
Proof By Lemmas 4.1 and 4.4, since $\mathcal{G}(G)$ is an open dense subset of $X^{V(G)}$ and $\mathcal{G}(G)^{c}$ has measure zero the set $\mathcal{R}(G) \cap \mathcal{G}(G)$ is non-empty, thus we choose $p^{\prime}$ to be a regular placement of $G$ in general position. Since $\left(G, p^{\prime}\right)$ is regular it follows that it is also independent.

Define $q:=\left.p^{\prime}\right|_{V(H)}$, then $(H, q)$ is in general position. As $(H, q) \subseteq\left(G, p^{\prime}\right)$ then by Proposition 4.10, $(H, q)$ is independent; furthermore as $H$ has at least $d+1$ vertices then $q$ is spanning. By Theorem 4.11, we have $|E(H)|=(\operatorname{dim} X)|V(H)|-$ $\operatorname{dim} \mathcal{F}(H, q)$. By Corollary 3.11 and Proposition 3.9, $\operatorname{dim} \mathcal{T}(q)=\operatorname{dim} \operatorname{Isom}(X)$, thus as $\mathcal{T}(q) \subset \mathcal{F}(H, q)$ we have the required inequality.

If $(G, p)$ is also isostatic then by Proposition 4.12, $|E(G)|=(\operatorname{dim} X)|V(G)|-$ $\operatorname{dim} \mathcal{T}(p)$. By Lemma 4.9(ii), $p$ is full, thus by Theorem 3.15, $\operatorname{dim} \mathcal{T}(p)=$ $\operatorname{dim} \operatorname{Isom}(X)$ as required.

\subsection{Proof of Theorem 1.1}

For a finite graph $G$, we say that a well-positioned framework $(G, p)$ is constant if there is a neighbourhood $\mathcal{N}(p)$ of $p$ in $X^{V(G)}$ such that $\mathcal{N}(p) \subset \mathcal{W}(G)$ and rank $d f_{G}(q)=\operatorname{rank} d f_{G}(p)$ for all $q \in \mathcal{N}(p)$. We shall denote $\mathcal{C}(G)$ to be the subset of $\mathcal{W}(G)$ of constant placements of $G$.

By Lemma 4.4, if a regular placement of a finite graph $G$ lies in the interior of $\mathcal{W}(G)$ then it will be constant. For Euclidean spaces $\mathcal{R}(G)=\mathcal{C}(G)$ as $\mathcal{R}(G)$ is an open dense subset of $X^{V(G)}$ (see [4, Sect. 3] for more details), however this does not hold for all normed spaces; see Example 4.17.

Lemma 4.14 Let $(G, p)$ a constant finite framework in $X$, then there exists an open neighbourhood $U \subset X^{V(G)}$ of $p$ such that $f_{G}^{-1}\left[f_{G}(p)\right] \cap U$ is a $C^{1}$-manifold with tangent space $\mathcal{F}(G, p)$ at $p$ and $\mathcal{O}_{p} \cap U$ is a $C^{1}$-submanifold of $f_{G}^{-1}\left[f_{G}(p)\right] \cap U$.

Proof Since $(G, p)$ is constant $p$ is an interior point of $\mathcal{W}(G)$, so by Proposition 4.2 and Lemma $4.3, f_{G}$ is $C^{1}$-differentiable with constant rank on an open neighbourhood of $p$ in $X^{V(G)}$. By the Constant Rank Theorem ([2, Thm. 2.5.15]) there exists an open neighbourhood $U \subset X^{V(G)}$ of $p$ such that $f_{G}^{-1}\left[f_{G}(p)\right] \cap U$ is a $C^{1}$-manifold with tangent space $\operatorname{ker} d f_{G}(p)=\mathcal{F}(G, p)$ at $p$.

By Theorem 3.6, $\mathcal{O}_{p}$ is a smooth manifold. As $\mathcal{O}_{p} \cap U \subseteq f_{G}^{-1}\left[f_{G}(p)\right] \cap$ $U \subseteq X^{V(G)}$ and both are $C^{1}$-submanifolds of $X^{V(G)}$ then the inclusion map $\mathcal{O}_{p} \cap U \hookrightarrow f_{G}^{-1}\left[f_{G}(p)\right] \cap U$ is a $C^{1}$-embedding, thus $\mathcal{O}_{p} \cap U$ is a $C^{1}$-submanifold of $f_{G}^{-1}\left[f_{G}(p)\right] \cap U$.

We are now ready to prove Theorem 1.1 .

Proof of Theorem 1.1 By Lemma 4.14, $\mathcal{O}_{p} \cap U$ is a $C^{1}$-submanifold of $f_{G}^{-1}\left[f_{G}(p)\right] \cap U$ for some open neighbourhood $U$ of $p$. As manifolds are locally path-connected we may assume we chose $U$ small enough such that $f_{G}^{-1}\left[f_{G}(p)\right] \cap U$ and $\mathcal{O}_{p} \cap U$ are path-connected. 
(Infinitesimal rigidity $\Leftrightarrow$ Local rigidity): Since $\mathcal{O}_{p} \cap U$ is a $C^{1}$-submanifold of $f_{G}^{-1}\left[f_{G}(p)\right] \cap U$ we have

$$
\begin{aligned}
& f_{G}^{-1}\left[f_{G}(p)\right] \cap U^{\prime}=\mathcal{O}_{p} \cap U^{\prime} \text { for some open neighbourhood } U^{\prime} \subseteq U \text { of } p \\
\Longleftrightarrow \quad & T_{p}\left(f_{G}^{-1}\left[f_{G}(p)\right] \cap U\right)=T_{p}\left(\mathcal{O}_{p} \cap U\right) \\
\Longleftrightarrow \quad & \mathcal{F}(G, p)=\mathcal{T}(p) ;
\end{aligned}
$$

this is equivalent to saying $(G, p)$ is infinitesimally rigid if and only if $(G, p)$ is locally rigid.

(Continuous rigidity $\Rightarrow$ Local rigidity): Suppose $(G, p)$ is continuously rigid. Choose $q \in f_{G}^{-1}\left[f_{G}(p)\right] \cap U$, then there exists a continuous path from $p$ to $q$ in $f_{G}^{-1}\left[f_{G}(p)\right] \cap U$. This implies that we may define a finite flex $\alpha$ of $(G, p)$ such that $\alpha\left(t_{0}\right)=q$ for some $t_{0} \in(-\delta, \delta)$. Since $(G, p)$ is continuously rigid then $\alpha$ is trivial and thus a continuous path in $\mathcal{O}_{p}$. It now follows $q \in \mathcal{O}_{p} \cap U$ as required.

(Local rigidity $\Rightarrow$ Continuous rigidity): Suppose $(G, p)$ is locally rigid, then there exists $\varepsilon>0$ such that $B_{\varepsilon}(p) \subset U$ (the open ball with respect to the normed space $\left.\left(X^{V(G)},\|\cdot\|_{V(G)}\right)\right)$ and $f_{G}^{-1}\left[f_{G}(p)\right] \cap B_{\varepsilon}(p)=\mathcal{O}_{p} \cap B_{\varepsilon}(p)$. First note that both $f_{G}^{-1}\left[f_{G}(p)\right]$ and $\mathcal{O}_{p}$ are invariant under $\operatorname{Isom}(X)$. Choose any $q \in \mathcal{O}_{p}$, then there exists $g \in \operatorname{Isom}(X)$ such that $g \cdot p=q$. We now note that

$$
\begin{aligned}
\mathcal{O}_{p} \cap B_{\varepsilon}(q) & =g \cdots\left(\mathcal{O}_{p} \cap B_{\varepsilon}(p)\right)=g \cdots\left(f_{G}^{-1}\left[f_{G}(p)\right] \cap B_{\varepsilon}(p)\right) \\
& =f_{G}^{-1}\left[f_{G}(p)\right] \cap B_{\varepsilon}(q) .
\end{aligned}
$$

As this holds for all $q \in \mathcal{O}_{p}$ then $\mathcal{O}_{p}$ is open in $f_{G}^{-1}\left[f_{G}(p)\right]$. By Lemma 3.4, $\mathcal{O}_{p}$ is closed in $X^{V(G)}$, thus $\mathcal{O}_{p}$ is clopen in $f_{G}^{-1}\left[f_{G}(p)\right]$.

Define $f_{G}^{-1}\left[f_{G}(p)\right]^{\Gamma}$ to be the path-connected component of $f_{G}^{-1}\left[f_{G}(p)\right]$ that contains $p$ with the subspace topology, then the only clopen set in $f_{G}^{-1}\left[f_{G}(p)\right]^{\Gamma}$ is itself. Define $\mathcal{O}_{p}^{\Gamma}:=\mathcal{O}_{p} \cap f_{G}^{-1}\left[f_{G}(p)\right]^{\Gamma}$, then $\mathcal{O}_{p}^{\Gamma}$ is clopen since $\mathcal{O}_{p}$ is clopen. This implies that $\mathcal{O}_{p}^{\Gamma}=f_{G}^{-1}\left[f_{G}(p)\right]^{\Gamma}$ and so any finite flex $\alpha$ lies in $\mathcal{O}_{p}$.

\subsection{Examples and Non-examples}

Example 4.15 Suppose $G$ is any finite graph and $\operatorname{smooth}(X)$ is an open subset of $X$ (most standard normed spaces fit this criteria, an example would be any $\ell_{q}^{d}$ space). We note $\mathcal{W}(G)$ will be an open subset of $X^{V(G)}$ and so by Lemma $4.4, \mathcal{R}(G)$ will be an open subset of $X^{V(G)}$. It now follows that every regular placement will be constant, thus by Theorem 1.1, if $(G, p)$ is infinitesimally rigid then it will be continuously and locally rigid also. Further, following a similar method to Lemma 4.4, we note that $\mathcal{C}(G)$ will also be an open dense subset of $X^{V(G)}$.

Example 4.16 For any finite graph $G$ and $q \in(1, \infty)$, it follows from the proof of [18, Lem. 2.7] that all constant placements of $G$ in $\ell_{q}^{d}$ are also regular. 
Example 4.17 For any $d$-dimensional real linear space $X$ and finite set $F \subset X^{*}$, we may define the polyhedral norm

$$
\|x\|_{F}:=\max _{f \in F}|f(x)| .
$$

By [15, Prop. 6], all well-positioned placements of finite graphs are constant, thus by Theorem 1.1, finite and infinitesimal rigidity are equivalent for any well-positioned finite framework; see [15, Thm. 7] for an alternative proof of the equivalence of infinitesimal rigidity and continuous rigidity.

Not all constant placements are regular however; in fact, $\mathcal{R}(G) \neq \mathcal{C}(G)$ if and only if $G$ contains a cycle. To see this, we first choose $x, y \in \operatorname{smooth}(X) \operatorname{such}$ that $\varphi(x), \varphi(y)$ are linearly independent. Let $G$ be a graph with vertices $\left\{v_{1}, \ldots, v_{n}\right\}$ and a cycle $C$; by reordering we may assume $v_{1} v_{n}$ is an edge of $C$. Define $p$ be the placement of $G$ with $p_{v_{i}}=i x$ for all $i=1, \ldots, n$, and $q$ be the placement of $G$ where $q_{v_{i}}=i x$ for all $i=1, \ldots, n$ and $q_{v_{n}}=\varepsilon y+x$, with $\varepsilon>0$ chosen such that $\varphi\left(q_{v_{n}}-q_{v_{i}}\right)$ is a scalar multiple of $\varphi(x)$ for all $i \geq 2$. Since both $p, q$ are well-positioned they are also constant. By direct calculation, we note that

$$
\operatorname{rank} d f_{G}(p)=\operatorname{rank} d f_{G \backslash\left\{v_{1} v_{n}\right\}}(q)=|E(G)|-1
$$

as they are both connected and all edges have the same support functional. If we choose $u \in X^{V(G)}$ with $u_{v_{n}} \in \operatorname{ker} \varphi(x) \backslash\{0\}$ and $u_{v_{i}}=0$ otherwise, then $u \in$ $\mathcal{F}\left(G \backslash\left\{v_{1}, v_{n}\right\}, q\right) \backslash \mathcal{F}(G, q)$. This implies rank $d f_{G}(q)>|E(G)|-1$, thus $p$ is a constant placement of $G$ that is not regular.

Example 4.18 Let $(G, p)$ be a finite infinitesimally rigid, full and constant framework in a 2-dimensional normed space $X$. Further, suppose there exist distinct $v_{1}, v_{2} \in$ $V(G)$ such that $y:=\frac{p_{v_{1}}-p_{v_{2}}}{\left\|p_{v_{1}}-p_{v_{2}}\right\|} \in \operatorname{smooth}(X)$. We now define the framework $\left(G^{\prime}, p^{\prime}\right)$, where $G^{\prime}$ is the graph with

$$
V\left(G^{\prime}\right):=V(G) \cup\left\{v_{0}\right\}, \quad E\left(G^{\prime}\right):=E(G) \cup\left\{v_{0} v_{1}, v_{0} v_{2}\right\},
$$

and $p^{\prime}$ is the placement where

$$
p_{v}^{\prime}:=p_{v}, \quad p_{v_{0}}^{\prime}:=\frac{p_{v_{1}}-p_{v_{2}}}{2}
$$

By choosing $u$ to be the flex with $u_{v_{0}} \in \operatorname{ker} \varphi(y)$ and $u_{v}=0$ for all $v \neq v_{0}$, we note that $\left(G^{\prime}, p^{\prime}\right)$ is infinitesimally flexible.

Consider $\varphi(y)^{-1}[1]$ with respect to the topology of $S_{1}^{*}[0]$. We now have two possibilities (Fig. 1):

(i) If $y$ lies in the interior of $\varphi(y)^{-1}[1]$, then $\left(G^{\prime}, p^{\prime}\right)$ is constant.

(ii) If $y$ lies on the boundary of $\varphi(y)^{-1}[1]$, then $\left(G^{\prime}, p^{\prime}\right)$ is not constant.

If (i) holds, by Theorem 1.1, $\left(G^{\prime}, p^{\prime}\right)$ is continuously and locally flexible also. Suppose (ii) and choose a sufficiently close framework $\left(G^{\prime}, q^{\prime}\right)$ such that $f_{G^{\prime}}\left(p^{\prime}\right)=f_{G^{\prime}}\left(q^{\prime}\right)$. 

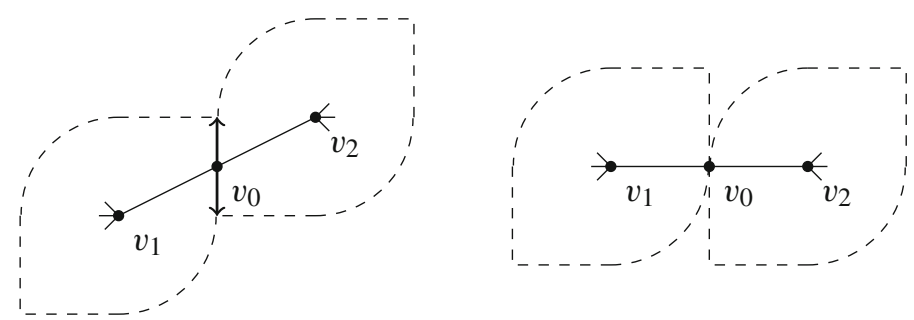

Fig. 1 Two possible placements of the vertices $v_{0}, v_{1}, v_{2}$ of $\left(G^{\prime}, p^{\prime}\right)$ as defined in Example 4.18. The dotted lines represent $S_{r}\left[p_{v_{1}}\right]$ and $S_{r}\left[p_{v_{2}}\right]$ for $r:=\frac{1}{2}\left\|p_{v_{1}}-p_{v_{2}}\right\|$. For the left placement, $v_{0}$ can be moved as indicated by the arrows, while for the right placement, $v_{0}$ can be seen to be fixed

By applying isometries we may assume $q_{v}^{\prime}=p_{v}^{\prime}$ for all $v \in V(G)$ as $(G, p)$ is locally rigid. By [21, Prop. 31], either $q^{\prime}=p^{\prime}$ or the line

$$
L:=\left[\frac{q_{v_{0}}^{\prime}-q_{v_{1}}^{\prime}}{\left\|q_{v_{0}}^{\prime}-q_{v_{1}}^{\prime}\right\|}, \frac{q_{v_{2}}^{\prime}-q_{v_{0}}^{\prime}}{\left\|q_{v_{2}}^{\prime}-q_{v_{0}}^{\prime}\right\|}\right]
$$

is contained in $S_{1}[0]$. If the latter holds then $y$ lies in the interior of $L$, thus $q^{\prime}=p^{\prime}$ and $\left(G^{\prime}, p^{\prime}\right)$ is locally rigid. It now follows from the proof of Theorem 1.1 that $\left(G^{\prime}, p^{\prime}\right)$ is continuously rigid. As $\left(G^{\prime}, p^{\prime}\right)$ is not constant this does not contradict Theorem 1.1.

In the following example, we shall construct a normed space that contains a framework that is locally flexible, continuously rigid and not constant. We shall denote the standard Euclidean norm of $\mathbb{R}^{2}$ by $\|\cdot\|_{2}$.

Example 4.19 Define for each $n \in \mathbb{N}$ the linear functions $f_{n}, g_{n}: \mathbb{R}^{2} \rightarrow \mathbb{R}$, where for any $r>0$ and $\theta \in(-\pi, \pi]$,

$$
\begin{aligned}
f_{n}(r \cos \theta, r \sin \theta) & :=\left(\frac{\cos \left(\frac{3 \pi}{2^{n+2}}\right)}{\cos \frac{\pi}{2^{n+2}}}\right) r \cos \theta+\left(\frac{\sin \left(\frac{3 \pi}{2^{n+2}}\right)}{\cos \frac{\pi}{2^{n+2}}}\right) r \sin \theta \\
& =\frac{r \cos \left(\frac{3 \pi}{2^{n+2}}-\theta\right)}{\cos \frac{\pi}{2^{n+2}}}, \\
g_{n}(r \cos \theta, r \sin \theta): & =\left(\frac{\cos \left(\frac{3 \pi}{2^{n+2}}\right)}{\cos \frac{\pi}{2^{n+2}}}\right) r \cos \theta-\left(\frac{\sin \left(\frac{3 \pi}{2^{n+2}}\right)}{\cos \frac{\pi}{2^{n+2}}}\right) r \sin \theta \\
& =\frac{r \cos \left(\frac{3 \pi}{2^{n+2}}+\theta\right)}{\cos \frac{\pi}{2^{n+2}}} .
\end{aligned}
$$

We now define the normed space $X:=\left(\mathbb{R}^{2},\|\cdot\|\right)$, where for all $(x, y) \in X$,

$$
\|(x, y)\|:=\sup \left\{\|(x, y)\|_{2},\left|f_{n}(x, y)\right|,\left|g_{n}(x, y)\right|: n \geq 2\right\}
$$



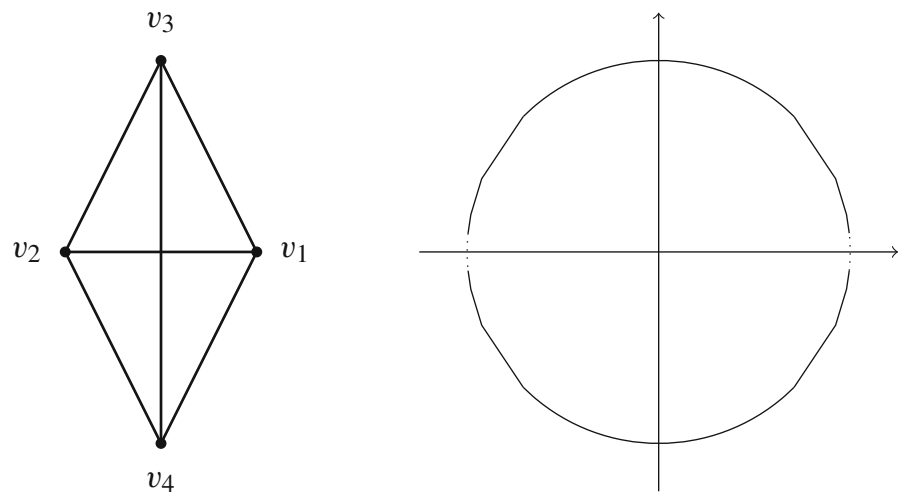

Fig. 2 The framework $(G, p)$ (left) and the unit ball of $X$ (right) as described in Example 4.19. The unit ball is obtained by "shaving pieces off" the unit disc

or equivalently,

$$
\|(x, y)\|:= \begin{cases}\left|f_{n}(x, y)\right| & \text { if } \frac{\pi}{2^{n+1}} \leq \tan ^{-1}\left(\frac{y}{x}\right) \leq \frac{\pi}{2^{n}} \text { for some } n \geq 2 \\ \left|g_{n}(x, y)\right| & \text { if } \frac{-\pi}{2^{n}} \leq \tan ^{-1}\left(\frac{y}{x}\right) \leq \frac{-\pi}{2^{n+1}} \text { for some } n \geq 2 \\ \|(x, y)\|_{2} & \text { otherwise }\end{cases}
$$

The norm $\|\cdot\|$ has been defined so that $S_{1}[0]$ is formed from the unit circle by replacing the arc from $(-1)^{j} \pi / 2^{n}+k \pi$ to $(-1)^{j} \pi / 2^{n+1}+k \pi$ by a straight line between the two points for each $j, k \in\{0,1\}$ and $n \geq 2$; see Fig. 2 .

We note that for any $(x, y)=(r \cos \theta, r \sin \theta),(x, y) \in \operatorname{smooth}(X)$ if and only if $r \neq 0$ and $\theta \neq \pm \pi / 2^{n}$ or $\pm \pi / 2^{n}+\pi$ for any $n \geq 2$; further, if $(x, y)$ is smooth then,

$$
\varphi(x, y)= \begin{cases}f_{n} & \text { if } \frac{\pi}{2^{n+1}}<\theta<\frac{\pi}{2^{n}} \text { for some } n \geq 2, \\ g_{n} & \text { if } \frac{-\pi}{2^{n}}<\theta<\frac{-\pi}{2^{n+1}} \text { for some } n \geq 2, \\ -f_{n} & \text { if } \frac{\pi}{2^{n+1}}+\pi<\theta<\frac{\pi}{2^{n}}+\pi \text { for some } n \geq 2, \\ -g_{n} & \text { if } \frac{-\pi}{2^{n}}+\pi<\theta<\frac{-\pi}{2^{n+1}}+\pi \text { for some } n \geq 2, \\ (a, b) \mapsto a x+b y & \text { otherwise. }\end{cases}
$$

Let $G$ be the complete graph on $v_{1}, v_{2}, v_{3}, v_{4}$ and $p$ the well-positioned placement of $G$ in $X$ with

$$
p_{v_{1}}=(1,0), \quad p_{v_{2}}=(-1,0), \quad p_{v_{3}}=(0,2), \quad p_{v_{4}}=(0,-2) .
$$

For $\theta \in\left(-\tan ^{-1}(1 / 3), \tan ^{-1}(1 / 3)\right)$, denote by $R(\theta) \in \operatorname{Isom}^{\operatorname{Lin}}(X)$ the anticlockwise rotation around the origin by an angle $\theta$. We note that $R(\theta)$ is an isometry of $X$ if and only if $\theta=0$, however $f_{G}(R(\theta) \cdot p)=f_{G}(p)$ if and only if either $\theta=0, \theta= \pm \pi / 2^{n}$ or $\pm \pi / 2^{n}+\pi$. As $R\left(\pi / 2^{n}\right) \cdot p \rightarrow p$ as $n \rightarrow \infty$ and $R\left(\pi / 2^{n}\right) \cdot p \in f_{G}^{-1}\left[f_{G}(p)\right] \backslash \mathcal{O}_{p}$ for all $n \in \mathbb{N},(G, p)$ is locally flexible. 
Given $\theta \in\left(-\tan ^{-1}(1 / 3), \tan ^{-1}(1 / 3)\right)$, we note that $(G, R(\theta) \cdot p)$ is wellpositioned if $\theta \neq \pi / 2^{n}$ for any $n \in \mathbb{N}$, and infinitesimally rigid if $\theta \neq 3 \pi / 2^{n}$ for any $n \in \mathbb{N}$ also. As $(G, p)$ is infinitesimally flexible and can be approximated by infinitesimally rigid placements, $(G, p)$ is not constant.

Let $\alpha$ be a non-trivial finite flex of $(G, p)$. Define $H:=\left(V(G), E(G) \backslash\left\{v_{1} v_{2}\right\}\right)$ and

$$
E:=\left\{q \in X^{V(G)}:\left\|q_{v}-q_{w}\right\|_{2}=\left\|p_{v}-p_{w}\right\|_{2} \text { for all } v w \in E(H)\right\}
$$

We observe that there exists an open neighbourhood $U \subset X^{V(G)}$ of $p$ such that

$$
f_{H}^{-1}\left[f_{H}(p)\right] \cap U=E \cap U,
$$

thus we may choose $0<\varepsilon<\delta$ such that $\alpha(t) \in U$ for all $t \in(-\varepsilon, \varepsilon)$, there exists $g_{t} \in \operatorname{Isom}\left(\mathbb{R}^{2},\|\cdot\|_{2}\right)$ such that $\alpha(t)=g_{t}$.p. As $\alpha$ is non-trivial, by applying translations and reflections, reparameterising, and choosing a sufficiently small value $\varepsilon>0$, we may assume that for all $t \in[0, \varepsilon)$,

$$
\begin{aligned}
& \alpha_{v_{1}}(t)=(\cos t, \sin t), \quad \alpha_{v_{2}}(t)=-\alpha_{v_{1}}(t), \quad \alpha_{v_{3}}(t)=(-2 \sin t, 2 \cos t), \\
& \alpha_{v_{4}}(t)=-\alpha_{v_{4}}(t) .
\end{aligned}
$$

Choose $n \in \mathbb{N}$ large enough such that $\pi / 2^{n} \in[0, \varepsilon)$. Then

$$
\left\|\alpha_{v_{1}}\left(\frac{3 \pi}{2^{n+2}}\right)-\alpha_{v_{2}}\left(\frac{3 \pi}{2^{n+2}}\right)\right\|=\frac{2 \cos \left(\frac{3 \pi}{2^{n+2}}-\frac{3 \pi}{2^{n+2}}\right)}{\cos \frac{\pi}{2^{n+2}}}=\frac{2}{\cos \frac{\pi}{2^{n+2}}}>2,
$$

contradicting that $\alpha$ is a finite flex of $(G, p)$. It now follows that $(G, p)$ is continuously rigid.

Remark 4.20 Example 4.19 is surprising, as for Euclidean normed spaces continuous rigidity and local rigidity are always equivalent (see [4, Prop. 1]). It is currently an open question to decide whether continuous rigidity and local rigidity are always equivalent for a given normed space.

\section{Flexibility of Small Frameworks and Stronger Bounds for $\mathcal{T}(p)$}

For any placement $p$ in $X$ we shall define $\mathcal{T}_{2}(p)$ to denote the space of trivial motions of $p$ in $\left(X,\|\cdot\|_{2}\right)$, the unique Euclidean space for $(X,\|\cdot\|)$ as defined in Lemma 2.4. If we refer to just $X$ we shall be referring to the general normed space $(X,\|\cdot\|)$.

Lemma 5.1 $\mathcal{T}(p)$ is a linear subspace of $\mathcal{T}_{2}(p)$.

Proof By Lemma 2.4, $\operatorname{Isom}(X,\|\cdot\|) \subseteq \operatorname{Isom}\left(X,\|\cdot\|_{2}\right)$. It now follows that $\mathcal{T}(p) \subseteq$ $\mathcal{T}_{2}(p)$. 
For Euclidean spaces we have the following equality for the dimension of the space of trivial motions for non-spanning placements.

Lemma 5.2 [9, Lem. 2.3.3] Let $(p, S)$ be a placement in $X$ where $d=\operatorname{dim} X$ and $n$ is the dimension of the affine span of $\left\{p_{v}: v \in S\right\}$. Then

$$
\operatorname{dim} \mathcal{T}_{2}(p)=\frac{(n+1)(2 d-n)}{2} .
$$

We now wish to obtain an upper and lower bound for the dimension of the space of trivial motions for non-spanning placements. To do this we shall first find an upperbound for when $|S|=2$ in non-Euclidean normed spaces and then use an inductive argument.

Lemma 5.3 Let $x_{0} \in X \backslash\{0\}$ and $\operatorname{dim} X=d$. Then the set

$$
\mathcal{O}\left(x_{0}\right):=\left\{T\left(x_{0}\right): T \in \operatorname{Isom}^{\operatorname{Lin}}(X)\right\}
$$

is a closed smooth submanifold of $X$; further $\operatorname{dim} \mathcal{O}\left(x_{0}\right)=d-1$ if and only if $X$ is Euclidean.

Proof Since $\operatorname{Isom}^{\operatorname{Lin}}(X)$ is compact then $\operatorname{Isom}^{\operatorname{Lin}}(X)$ gives rise to a proper Lie group action on $X$ by $x \mapsto T(x)$ for all $T \in \operatorname{Isom}^{\operatorname{Lin}}(X), x \in X$. As $\mathcal{O}\left(x_{0}\right)$ is the orbit of $x_{0}$ (with respect to $\operatorname{Isom}^{\operatorname{Lin}}(X)$ ) then by Lemma 3.1, $\mathcal{O}\left(x_{0}\right)$ is a closed smooth submanifold of $X$.

First suppose $X$ is Euclidean. By [27, Cor. 3.3.3], Isom ${ }^{\operatorname{Lin}}(X)$ acts transitively on $S_{\left\|x_{0}\right\|}[0]$, thus $\mathcal{O}\left(x_{0}\right)=S_{\left\|x_{0}\right\|}[0]$. As the unit sphere of a Euclidean space is the $d$-sphere and $S_{\left\|x_{0}\right\|}[0]=\left\|x_{0}\right\| S_{1}[0]$ we have $\operatorname{dim} \mathcal{O}\left(x_{0}\right)=d-1$.

Now suppose $\operatorname{dim} \mathcal{O}\left(x_{0}\right)=d-1$. If $d=1$ the result is immediate so assume $d>1$. The set $S_{\left\|x_{0}\right\|}[0]$ is a closed connected topological submanifold of $X$ with dimension $d-1$ as it is homeomorphic to the $d$-sphere. Since $\mathcal{O}\left(x_{0}\right) \subset S_{\left\|x_{0}\right\|}[0]$ then $\mathcal{O}\left(x_{0}\right)$ is a closed subset of $S_{\left\|x_{0}\right\|}[0]$. As $\operatorname{dim} \mathcal{O}\left(x_{0}\right)=\operatorname{dim} S_{\left\|x_{0}\right\|}[0]$ it follows from Brouwer's theorem for invariance of domain [20, Thm. 1.18] that the inclusion map $\mathcal{O}\left(x_{0}\right) \hookrightarrow S_{\left\|x_{0}\right\|}[0]$ is an open map and thus $\mathcal{O}\left(x_{0}\right)$ is an open subset of $S_{\left\|x_{0}\right\|}[0]$. As the only clopen non-empty subset of $S_{\left\|x_{0}\right\|}[0]$ is itself we have $\mathcal{O}\left(x_{0}\right)=S_{\left\|x_{0}\right\|}[0]$. This implies $\operatorname{Isom}^{\operatorname{Lin}}(X)$ acts transitively on $S_{\left\|x_{0}\right\|}[0]$, thus by [27, Cor. 3.3.5], $X$ is Euclidean.

Lemma 5.4 Let $(p, S)$ be a general positioned placement in a d-dimensional space $X$ where $|S|=2$. Then $\operatorname{dim} \mathcal{T}(p) \leq 2 d-1$ with equality if and only if $X$ is Euclidean.

Proof By Lemmas 5.1 and 5.2 it follows $\operatorname{dim} \mathcal{T}(p) \leq 2 d-1$ and $\operatorname{dim} \mathcal{T}(p)=2 d-1$ if $X$ is Euclidean and $p$ is in general position.

Now suppose $\operatorname{dim} \mathcal{T}(p)=2 d-1$. Let $S=\left\{v_{1}, v_{2}\right\}$, then without loss of generality we may assume $p_{v_{1}}=0$. If $d=1$ the result holds trivially so we may suppose $d>1$. As $p$ is in general position then $p_{v_{2}} \neq 0$. By Lemma 5.3, $\mathcal{O}\left(p_{v_{2}}\right)$ is a closed smooth submanifold of $X$. It follows from [2, Lem. 3.3.4] that we may identify the tangent space of $\mathcal{O}\left(p_{v_{2}}\right)$ at $p_{v_{2}}$ with a subspace of $X$; by calculation we see that the tangent 
space of $\mathcal{O}\left(p_{v_{2}}\right)$ at $p_{v_{2}}$ is $\{u \in X:(0, u) \in \mathcal{T}(p)\}$. Since $\operatorname{dim} \mathcal{T}(p)=d+(d-1)$ and the trivial motions generated by translations form a $d$-dimensional subspace then it follows $\mathcal{O}\left(p_{v_{2}}\right)$ has dimension $d-1$. By Lemma 5.3 the space $X$ is Euclidean as required.

Lemma 5.5 Let $(p, S)$ be a placement in a d-dimensional space $X$ and $\left\{p_{v}: v \in S\right\}$ have an affine span of dimension $k-1$ where $2 \leq k \leq d$. Suppose $\left(p^{\prime}, S^{\prime}\right)$ is a placement where $(p, S) \subset\left(p^{\prime}, S^{\prime}\right)$ and the dimension of the affine span of $\left\{p_{v}^{\prime}: v \in S^{\prime}\right\}$ is $k$, then

$$
\operatorname{dim} \mathcal{T}\left(p^{\prime}\right)-\operatorname{dim} \mathcal{T}(p) \leq d-k
$$

Proof Choose $T:=\left\{v_{0}, \ldots, v_{k-1}\right\}$ and $T^{\prime}:=T \cup\left\{v_{k}\right\}$ where $v_{i} \in S$ for $i \in$ $\{0, \ldots, k-1\}, v_{k} \in S^{\prime}$ and $p_{v_{0}}^{\prime}, \ldots, p_{v_{k}}^{\prime}$ have affine span with dimension $k$. Define the linear restriction map

$$
P: X^{T^{\prime}} \rightarrow X^{T},\left(x_{v_{i}}\right)_{i=0}^{k} \mapsto\left(x_{v_{i}}\right)_{i=0}^{k-1}
$$

and the placements $(q, T) \subset(p, S)$ and $\left(q^{\prime}, T^{\prime}\right) \subset\left(p^{\prime}, S^{\prime}\right)$. We note that $P\left(\mathcal{T}_{2}\left(q^{\prime}\right)\right) \subseteq$ $\mathcal{T}_{2}(q)$ and $P\left(\mathcal{T}\left(q^{\prime}\right)\right) \subseteq \mathcal{T}(q)$. Choose any $u \in \mathcal{T}(q)$, then by Corollary 3.7 there exists $h \in T_{l} \operatorname{Isom}(X)$ such that $u=h . q$. By Corollary 3.7, h. $q^{\prime} \in \mathcal{T}\left(q^{\prime}\right)$, thus as $P\left(h . q^{\prime}\right)=h . q$ we have $P\left(\mathcal{T}\left(q^{\prime}\right)\right)=\mathcal{T}(q)$. By a similar argument we can see that $P\left(\mathcal{T}_{2}\left(q^{\prime}\right)\right)=\mathcal{T}_{2}(q)$ also.

By the Rank-Nullity theorem applied to $\left.P\right|_{\mathcal{T}_{2}\left(q^{\prime}\right)}$ and Lemma 5.2

$$
\begin{aligned}
\left.\operatorname{dim} \operatorname{ker} P\right|_{\mathcal{T}_{2}\left(q^{\prime}\right)} & =\operatorname{dim} \mathcal{T}_{2}\left(q^{\prime}\right)-\operatorname{dim} \mathcal{T}_{2}(q)=\frac{(k+1)(2 d-k)}{2}-\frac{k(2 d-k+1)}{2} \\
& =d-k
\end{aligned}
$$

By Lemma 5.1 and the Rank-Nullity theorem applied to $\left.P\right|_{\mathcal{T}\left(q^{\prime}\right)}$

$$
\operatorname{dim} \mathcal{T}\left(q^{\prime}\right)-\operatorname{dim} \mathcal{T}(q)=\left.\operatorname{dim} \operatorname{ker} P\right|_{\mathcal{T}\left(q^{\prime}\right)} \leq\left.\operatorname{dim} \operatorname{ker} P\right|_{\mathcal{T}_{2}\left(q^{\prime}\right)}=d-k
$$

By Corollary $3.8($ ii $), \mathcal{T}(q) \cong \mathcal{T}(p)$ and $\mathcal{T}\left(q^{\prime}\right) \cong \mathcal{T}\left(p^{\prime}\right)$ and so the result follows.

Proposition 5.6 Let $(p, S)$ be a placement in a d-dimensional normed space $X$ where $\left\{p_{v}: v \in S\right\}$ has an affine span of dimension $1 \leq n \leq d$. Then

$$
\operatorname{dim} \mathcal{T}(p) \leq \frac{(n+1)(2 d-n)}{2}
$$

with equality if and only if $X$ is Euclidean.

Proof If $X$ is Euclidean then the result follows by Lemma 5.2.

Suppose $X$ is non-Euclidean. If $n=1$ then the result follows by Lemma 5.4 and Corollary 3.8 (ii). Let $n>1$ and suppose the theorem holds for all $m=1, \ldots, n-1$. Choose $v_{1}, \ldots, v_{n} \in S$ so that $p_{v_{1}}, \ldots, p_{v_{n}}$ are affinely independent and define $T:=$ 
$\left\{v_{1}, \ldots, v_{n}\right\}$. If we define $q:=\left.p\right|_{T}$ then $\left\{q_{v}: v=v_{1}, \ldots, v_{n}\right\}$ has an affine span with dimension $n-1$, thus by assumption $\operatorname{dim} \mathcal{T}(q)<n(2 d-n+1) / 2$. By Lemma 5.5 it follows that

$$
\operatorname{dim} \mathcal{T}(p) \leq \operatorname{dim} \mathcal{T}(q)+d-n<\frac{n(2 d-n+1)}{2}+d-n=\frac{(n+1)(2 d-n)}{2} .
$$

We define a framework $(G, p)$ in a $d$-dimensional normed space to be small if $|V(G)| \leq d+1$. The following is a well-known result for Euclidean spaces.

Proposition 5.7 Let $X$ be d-dimensional Euclidean space and $(G, p)$ be a small wellpositioned framework, then $(G, p)$ is isostatic if and only if $G$ is a complete graph and $p$ is in general position.

Proof $(\Rightarrow)$ If $(G, p)$ is isostatic it follows by Proposition 4.12 and Lemma 5.2 that $|E(G)|=|V(G)|(|V(G)|-1) / 2$, thus $G$ is a complete graph.

Suppose $p$ is not in general position, then there exist distinct vertices $v_{0}, \ldots, v_{n}, w$ $\in V(G)$ such that the affine span of $p_{v_{0}}, \ldots, p_{v_{n}}$ is $Y \subset X$, the affine span of $\left\{p_{v}: v \in V(G)\right\}$. Define for each $v \in V(G)$ the vector $u_{v}$ where $u_{v}=0$ if $v \neq w$ and $u_{w} \in\left(Y-p_{v_{0}}\right)^{\perp} \backslash\{0\}$, then $u:=\left(u_{v}\right)_{v \in V(G)} \in X^{V(G)}$ is an infinitesimal flex of $(G, p)$. If $u$ is trivial then by Corollary 3.7 there exists an affine map $g \in T_{\iota} \operatorname{Isom}(X)$ such that $g . p=u$. As $p_{v_{0}}, \ldots, p_{v_{n}}$ is an affine basis of $Y$ and $g\left(p_{v_{i}}\right)=0$ for all $i=0,1, \ldots, n$ then $g(y)=0$ for all $y \in Y$. However $g\left(p_{w}\right) \neq 0$ and so no such affine map $g$ may exist. This implies $u$ is a non-trivial flex which contradicts the infinitesimal rigidity of $(G, p)$.

$(\Leftarrow)$ This follows from $[9$, Thm. 2.4.1.d].

Using Theorem 3.15 we can now state our own result for small frameworks for non-Euclidean spaces.

Theorem 5.8 Let $(G, p)$ be a small well-positioned framework with in a d-dimensional non-Euclidean normed space $X$, then $(G, p)$ is infinitesimally flexible or $|V(G)|=1$.

Proof If $|V(G)|=1$ then $(G, p)$ is infinitesimally rigid by definition. Suppose $|V(G)| \geq 2$ and the affine span of $\left\{p_{v}: v \in V(G)\right\}$ has dimension $n$. Define the map $f: \mathbb{R} \rightarrow \mathbb{R}$ where

$$
f(x)=\frac{(x+1)(2 d-x)}{2} .
$$

We note that $f$ is increasing on the interval $[0, d-1]$ and $f(d-1)=f(d)$, thus it follows that $f(|V(G)|-1) \geq f(n)$. We note

$$
\begin{aligned}
|E(G)| & \leq \frac{|V(G)|(|V(G)|-1)}{2} \\
& =d|V(G)|-f(|V(G)|-1)
\end{aligned}
$$




$$
\begin{aligned}
& \leq d|V(G)|-f(n) \\
& <d|V(G)|-\operatorname{dim} \mathcal{T}(p) \text { by Lemma } 5.6
\end{aligned}
$$

thus by Theorem 4.11, $(G, p)$ is infinitesimally flexible.

Remark 5.9 (Final remark) We remark that combinatorial characterisations of infinitesimal rigidity have recently been obtained in some normed space contexts. However other forms of rigidity, such as redundant and global rigidity, have not yet been explored in general normed spaces.

Open Access This article is distributed under the terms of the Creative Commons Attribution 4.0 International License (http://creativecommons.org/licenses/by/4.0/), which permits unrestricted use, distribution, and reproduction in any medium, provided you give appropriate credit to the original author(s) and the source, provide a link to the Creative Commons license, and indicate if changes were made.

\section{References}

1. Abraham, R., Marsden, J.E.: Foundations of Mechanics, 2nd edn. Addison-Wesley, Reading (1978)

2. Abraham, R., Marsden, J.E., Raiţu, T.S.: Manifolds, Tensor Analysis, and Applications, 3rd edn. Springer, New York (2002)

3. Amir, D.: Characterizations of Inner Product Spaces. Operator Theory: Advances and Applications, vol. 20. Birkhäuser, Basel (1986)

4. Asimow, L., Roth, B.: The rigidity of graphs. Trans. Am. Math. Soc. 245, 279-289 (1978)

5. Asimow, L., Roth, B.: The rigidity of graphs II. J. Math. Anal. Appl. 68(1), 171-190 (1979)

6. Benyamini, Y., Lindenstrauss, J.: Geometric Nonlinear Functional Analysis, vol. I. American Mathematical Society Colloquium Publications, vol. 48. American Mathematics Society, Providence (2000)

7. Gluck, H.: Almost all simply connected closed surfaces are rigid. In: Geometric Topology. Lecture Notes in Mathematics, vol. 438, pp. 225-239. Springer, Berlin (1975)

8. Gortler, S.J., Thurston, D.P.: Characterizing the universal rigidity of generic frameworks. Discret. Comput. Geom. 51(4), 1017-1036 (2014)

9. Graver, J., Servatius, B., Servatius, H.: Combinatorial Rigidity Graduate Studies in Mathematics, vol. 2. American Mathematics Society, Providence (1993)

10. Hall, B.: Lie Groups, Lie Algebras, and Representations: An Elementary Introduction. Graduate Texts in Mathematics, vol. 222. Springer, New York (2003)

11. Jackson, B., Jordán, T.: The generic rank of body-bar-and-hinge frameworks. Eur. J. Combin. 31(2), 574-588 (2010)

12. Jordán, T.: Combinatorial rigidity: graphs and matroids in the theory of rigid frameworks. Technical report TR-2014-12. Egerváry Research Group, Budapest (2014)

13. Jordán, T., Kaszanitzky, V.E., Tanigawa, S.: Gain-sparsity and symmetry-forced rigidity in the plane. Discret. Comput. Geom. 55(2), 314-372 (2016)

14. Katoh, N., Tanigawa, S.: A proof of the molecular conjecture. Discret. Comput. Geom. 45(4), 647-700 (2011)

15. Kitson, D.: Finite and infinitesimal rigidity with polyhedral norms. Discret. Comput. Geom. 54(2), 390-411 (2015)

16. Kitson, D., Levene, R.H.: Graph rigidity for unitarily invariant matrix norms (2017). arXiv:1709.08967

17. Kitson, D., Power, S.C.: Infinitesimal rigidity for non-Euclidean bar-joint frameworks. Bull. Lond. Math. Soc. 46(4), 685-697 (2014)

18. Kitson, D., Power, S.C.: The rigidity of infinite graphs. Discret. Comput. Geom. 60(3), 531-557 (2018)

19. Kitson, D., Schulze, B.: Maxwell-Laman counts for bar-joint frameworks in normed spaces. Linear Algebra Appl. 481, 313-329 (2015)

20. Lee, J.M.: Manifolds and Differential Geometry. Graduate Studies in Mathematics, vol. 107. American Mathematical Society, Providence (2009) 
21. Martini, H., Swanepoel, K.J., Weiß, G.: The geometry of Minkowski spaces-a survey. Part I. Expo. Math. 19(2), 97-142 (2001)

22. Montgomery, D., Samelson, H.: Transformation groups of spheres. Ann. Math. 44(3), 454-470 (1943)

23. Nixon, A., Owen, J.C., Power, S.C.: Rigidity of frameworks supported on surfaces. SIAM J. Discret. Math. 26(4), 1733-1757 (2012)

24. Rockafellar, R.T.: Convex Analysis. Princeton Mathematical Series, vol. 28. Princeton University Press, Princeton (1970)

25. Schulze, B., Tanigawa, S.: Infinitesimal rigidity of symmetric bar-joint frameworks. SIAM J. Discret. Math. 29(3), 1259-1286 (2015)

26. Tanigawa, S.: Sufficient conditions for the global rigidity of graphs. J. Comb. Theory Ser. B 113, 123-140 (2015)

27. Thompson, A.C.: Minkowski Geometry. Encyclopedia of Mathematics and its Applications, vol. 63. Cambridge University Press, Cambridge (1996)

Publisher's Note Springer Nature remains neutral with regard to jurisdictional claims in published maps and institutional affiliations. 